\title{
Productivity of Edalat-Potts Exact Arithmetic in Constructive Type Theory*
}

\author{
Milad Niqui \\ Institute for Computing and Information Sciences, Radboud University Nijmegen, \\ Toernooiveld 1, 6525 ED Nijmegen, The Netherlands \\ M.Niqui@cs.ru.nl
}

\begin{abstract}
In this work we focus on a formalisation of the algorithms of lazy exact arithmetic à la Edalat-Potts in type theory. We choose the constructive type theory extended with coinductive types as our formal verification tool. We show examples of how infinite objects such as streams and expression trees can be formalised as coinductive types. We study the type theoretic notion of productivity which ensures the infiniteness of the outcome of the algorithms on infinite objects. Syntactical methods are not always strong enough to ensure the productivity. However, if some information about the complexity of a function is provided, one may be able to show the productivity of that function. In the case of the normalisation algorithm we show that such information can be obtained from the choice of real number representation that is used to represent the input and the output. Furthermore, we show how to employ this semantic information to formalise a restricted case of normalisation algorithm in our type theory.
\end{abstract}

\section{Introduction}

In the exact arithmetic approach to numerical computations the emphasis lies on the precision of the outcome of the computation. Due to this the algorithms of exact arithmetic are suitable objects for applying formal methods. Both of the fields of exact arithmetic and formal methods benefit from the verification of exact arithmetic by means of formal tools such as theorem provers or proof assistants. On one hand such formalisations often expose some rarely considered subtleties in the object of the formalisation. On the other

* This work was partially supported by the Netherlands Organisation for Scientific Research (NWO). 
hand formalising exact arithmetic provides a serious test to assess the expressiveness of the formal framework that is chosen for the formalisation. This is especially the case if one uses the approaches to the exact arithmetic whose formalisation requires a complex type system, such as the lazy exact arithmetic à la Edalat and Potts [12].

Formalising Edalat-Potts algorithms requires a type theory that is enriched with infinite objects. This is because the Normalisation Algorithm (NAlg) — which is the core of Edalat-Potts' lazy exact arithmetic — has infinite objects, namely streams and expression trees, as its input and output. Streams and expression trees are both relatively simple infinite objects and therefore they can be formalised as function types in simple type theories. However, such formalisations require modifying NAlg in order to fit in the said type system. Usually this means that the formalised version of NAlg is not a lazy algorithm anymore; in that it cannot operate on lazy objects as input and it does not output lazy objects. Rather, it performs the computation in a sequential manner.

In our work we intend to formalise the NAlg while keeping its lazy nature, because in our view laziness is an important characteristic of NAlg. For instance, lazy evaluation allows several copies of NAlg to be composed in order to evaluate complex algebraic expressions involving real numbers, while limiting the use of computer resources. Thus in order to keep this lazy nature we choose a type theory extended with coinductive types. We base our work on Martin-Löf constructive type theory which is a programming language where proofs and computations coexist [19], [25]. We present the simplest way of adding coinductive types to Martin-Löf type theory by directly formalising the rules for weakly final coalgebras of a restricted class of polynomial functors as typing judgements. This setting will be enough for presenting the types of streams and expression trees which are used in the statement of NAlg. The main part of the paper will deal with formalising NAlg in this setting.

The structure of the paper is as follows. In Section 2 we present the rules for enriching constructive type theory with simple independent coinductive types. In Section 3 we introduce the coinductive type of streams and expression trees over some given sets. This, together with the basics of the theory of Möbius maps and digit sets, enables us to present the informal version of NAlg in Section 4. This informal version is given in a form which is implementable in an ordinary functional programming language such as Haskell. However, it is not a valid definition in constructive type theory. This is due to the fact that NAlg is a partial function. In Section 5 we introduce the notion of productivity which provides a semantics for partiality on infinite objects. In Section 6 we present a semantical treatment of productivity of NAlg based on the topological properties of Möbius maps. In Section 7 we show how one can formalise the NAlg in coinductive type theory using the semantical proof of productivity. In Section 8 we present a general analysis of the methodology used. In Section 9 we point out a more syntactical type theoretic approach which requires stronger machinery for coinductive types.

\section{Coinductive Types}

Coinductive types are added to type theory for dealing with infinite objects [21]. Recall that following Martin-Löf's methodology, in order to present the rules for a type, one should present the formation, introduction, elimination and equality rules [19]. Here we 
use this approach to define coinductive types for polynomial functors. Such coinductive types are all that is needed for formalising NAlg.

We base our works on the intensional version of Martin-Löf type theory [25] which we denote by MTT. In the Martin-Löf's setting, the formation rule is originally a set formation rule. However, we work in an ambient category $\mathcal{C}$ without any constraints. The formation rule will be modified to the formation of the objects of $\mathcal{C}$. For a symbol $t$ to be a type, it should be an object of $\mathcal{C}$. We fix a distinguished symbol $s$. Then ' $t$ is a type' can be written as $t: s$. An endofunctor on $\mathcal{C}$ will correspond to a type operator. We present the method of construction for a very simple class of polynomial functors, ${ }^{1}$ namely those of the form $\mathrm{F}(X)=A_{0}+A_{1} \times X+\cdots+A_{n} \times X^{n}$.

Below we present the rules of coinductive types. The method of construction will be given as a type constructor symbol $v$ for polynomial functor $\mathrm{F}$. This method of construction is characterised by the following rules and produces the coinductive type $v \mathrm{~F}$ :

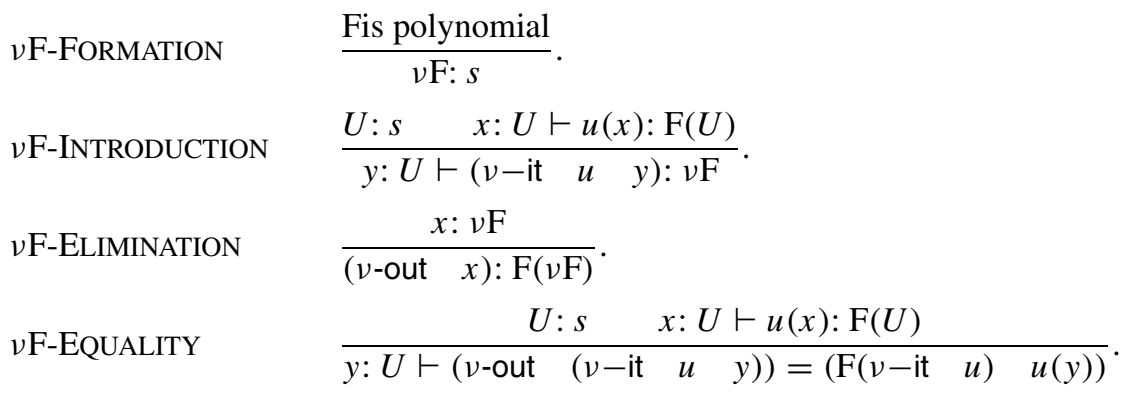

The rule $v$ F-EQUALITY speaks about the intensional equality between terms. This means that it can be considered as a reduction or conversion rule. This way we can present the following rule:

$$
\nu \text { F-REDUCTION } \quad(\nu \text {-out } \quad(v-\text { it } \quad u \quad y)) \sim(\mathrm{F}(\nu-\text { it } \quad u) \quad u(y)) \text {. }
$$

The rules are inspired by the coiteration scheme of the final coalgebra of $\mathrm{F}$ which is simply spelling out the existence of the coiterator, i.e., the map $v$-it $(u)$ that makes the following diagram commute:

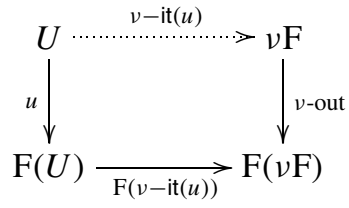

The alternative schemes that can be used for defining functions into a final coalgebra, such as the corecursion scheme or the dual of course-of-value iteration scheme lead to similar introduction and reduction rules [23, Section 4.4].

At this point we are able to use $v$-FORMATION to introduce the coinductive types. Evidently we only need to know whether the given functor is polynomial. Since we

\footnotetext{
${ }^{1}$ The reason for considering such a restricted form is that it makes the presentation of constructors (Definition 2.1) easier.
} 
are only interested in simple independent types we can rely on the fact that polynomial functors can be completely specified in terms of their constructors. ${ }^{2}$ In this definition $\mathrm{in}_{i}^{(n)}: X_{i} \longrightarrow X_{0}+\cdots+X_{n}$ is the $i$ th coprojection of the $n$-ary coproduct.

Definition 2.1 (Constructor). Let $\mathrm{F}(X)=A_{0}+A_{1} \times X+\cdots+A_{n} \times X^{n}$ be a polynomial functor with final coalgebra $\langle\nu \mathrm{F}, v$-out $\rangle$. We define the $i$ th constructor $(0 \leq i \leq n)$ of $\mathrm{F}$ to be the map con ${ }_{i}: A_{i} \times \nu \mathrm{F}^{i} \rightarrow \nu \mathrm{F}$ defined as

$$
\operatorname{con}_{i}:=v-\mathrm{it}(\mathrm{F}(\nu \text {-out })) \circ \mathrm{in}_{i}^{(n)},
$$

where $j(0)=0$ and $j(i)=1, i>0$.

Recall the general fact that the structural map of a final coalgebra is an isomorphism [28, Theorem 9.1]. Then we can use the above definition to obtain the characteristic property of constructors: if one constructs an object using a constructor and then destructs it using the structure map, one observes the original object. This phenomenon is captured in the following proposition.

Proposition 2.2. Let $\mathrm{F}(X)=A_{0}+A_{1} \times X+\cdots+A_{n} \times X^{n}$ be a polynomial functor with final coalgebra $\langle\nu \mathrm{F}, \nu$-out $\rangle$.

(i) For each $i(0 \leq i \leq n) \nu$-out $\circ \operatorname{con}_{i}=\mathrm{id}_{\mathrm{A}_{\mathrm{j}} \times \nu \mathrm{F}^{\mathrm{i}}}$.

(ii) $\left(\sum_{i=0}^{n} \operatorname{con}_{i}\right) \circ v$-out $=\mathrm{id}_{\nu \mathrm{F}}$.

This means that we can reconstruct a final coalgebra from its constructors. In terms of our $\nu$ F-FORMATION rule it means that existence of the constructors is enough for formation of a coinductive type because it implies that $\mathrm{F}$ is polynomial. This approach is taken in Coq for defining coinductive types [16].

After forming a coinductive type using $v$-FORMATION one can use the remaining rules for defining functions on infinite objects and proving the properties of these functions. However, the intensional equality given by $v$ F-EQUALITY is usually not strong enough for observational properties of infinite object. For example, in the case of streams many useful properties have to be stated and proven in terms of the bisimulation equivalence and that requires working with setoids [5, Section 3.3]. In this paper we are not concerned with this as we are only interested in the productivity of the NAlg. In future work when we deal with the correctness of NAlg we have to take this into account.

Furthermore, in this paper we do not consider the issue of consistency of the above extension of MTT. As in our extension we restricted ourselves to simple polynomial types, the resulting type theory is a simplified case of other more complex extensions of MTT which are consistent (see, e.g., [22]).

\footnotetext{
${ }^{2}$ In the presence of dependent types, a complete set of rules requires presenting a data type for a more general form of polynomial functors [17].
} 


\section{Streams and Expression Trees}

We introduce the coinductive types that are used in NAlg. Let $A, B$ be two sets and define the following functors:

$$
\mathrm{F}_{\mathrm{A}}(X)=A \times X, \quad \mathrm{~F}_{\mathrm{AB}}(X)=A \times X+B \times X^{2} .
$$

The above functors have final coalgebras in the category of sets. The carrier of the final coalgebra of $\mathrm{F}_{\mathrm{A}}$ is just the set $A^{\omega}$ of stream of elements of $A$. It is well known that the set of streams with a structure map $\langle\mathrm{hd}, \mathrm{t}\rangle: A^{\omega} \rightarrow A \times A^{\omega}$ is a final coalgebra for $\mathrm{F}_{\mathrm{A}}$ [28]. The constructor of the final coalgebra of streams of $A$ is cons: $A \rightarrow A^{\omega}$ which prepends an element to the beginning of a stream. We write $a$ : $\alpha$ for $\operatorname{cons}(a, \alpha)$. Thus, by presenting the above constructor we can form coinductive type of streams using $\nu \mathrm{F}_{\mathrm{A}}$-FORMATION.

The carrier of the final coalgebra of $\mathrm{F}_{\mathrm{AB}}$ is the set $\mathbb{E}(A, B)$ of expression trees with $A$-elements and $B$-operations. These are infinite trees (i.e., every branch is infinite) in which every node has one or two children. A unary node is an element of $A$ and a binary node is an element of $B$. An expression tree has no leaves. The structure map can be defined as

$$
\operatorname{psupp}_{\mathrm{A}, \mathrm{B}}(\theta):= \begin{cases}\operatorname{inl}\left(\left\langle a, \theta^{\prime}\right\rangle\right) & \text { if } \theta \text { has root } a \text { and child } \theta^{\prime}, \\ \operatorname{inr}\left(\left\langle b, \theta^{\prime}, \theta^{\prime \prime}\right\rangle\right) & \text { if } \theta \text { has root } b \text { and children } \theta^{\prime}, \theta^{\prime \prime} .\end{cases}
$$

One can prove that the pair $\left\langle\mathbb{E}(A, B)\right.$, psupp $\left._{\mathrm{A}, \mathrm{B}}\right\rangle$ is the final $\mathrm{F}_{\mathrm{AB}}$-coalgebra in the category of sets [23, Example 4.2.5].

Definition 2.1 gives us the two constructors of $\mathrm{F}_{\mathrm{AB}}$ which have the following types:

ucons: $A \times \mathbb{E}(A, B) \rightarrow \mathbb{E}(A, B)$,

bcons: $B \times \mathbb{E}(A, B)^{2} \rightarrow \mathbb{E}(A, B)$.

We write $\langle\langle a ; \theta\rangle\rangle$ and $\left\langle\left\langle b ; \theta, \theta^{\prime}\right\rangle\right\rangle$ respectively for ucons $(a, \theta)$ and bcons $\left(b, \theta, \theta^{\prime}\right)$. Thus, by presenting the above constructors we can form the coinductive type of expression trees using $v \mathrm{~F}_{\mathrm{AB}}$-FORMATION.

\section{The Normalisation Algorithm}

The Normalisation Algorithm (NAlg) of Edalat and Potts is an algorithm that transforms an expression tree of matrices and tensors to a stream of matrices that represents a real number. This algorithm is presented by Edalat and Potts as a unified way of computing elementary functions on real numbers, by encoding real functions as families of expression trees [12], [26], [13]. We can present NAlg using the constructors for expression trees and streams. However, first we need to define the sets of matrices and tensors that are used in NAlg.

$$
\begin{aligned}
& \text { Let } \mathbb{R}^{+}=(0,+\infty) \text { and } \mathbb{R}^{\star}:=\mathbb{R}^{+} \cup\{0,+\infty\} \text {. A Möbius map is a map } \\
& \mu(x):=\frac{a x+b}{c x+d},
\end{aligned}
$$


where $a, b, c, d \in \mathbb{Z}$. A refining Möbius map is a nonsingular Möbius map (i.e., $a d-b c \neq$ 0 ) that maps $\mathbb{R}^{\star}$ to itself. ${ }^{3}$ Note that a $2 \times 2$ matrix is just another notation for a Möbius map.

Similarly, a quadratic map is a map

$$
\xi\langle x, y\rangle:=\frac{a x y+b x+c y+d}{e x y+f x+g y+h},
$$

with $a, b, c, d, e, f, g \in Z$ and can be denoted by its $2 \times 2 \times 2$ tensor of coefficients. This tensor can in turn be denoted by a $2 \times 4$ matrix:

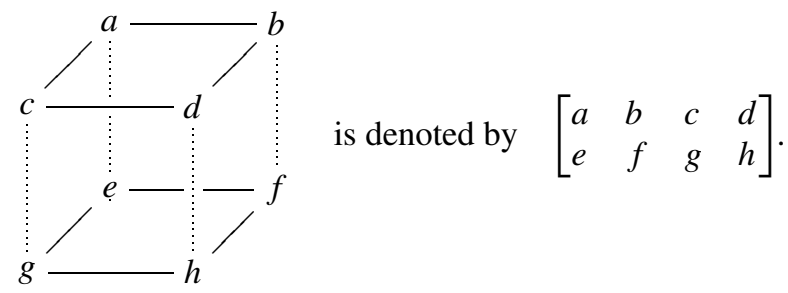

In the rest of the paper by tensor we mean the quadratic map with the nodes of the above cube as coefficients. If all of the six matrices that constitute the faces of the above cube are nonsingular then we shall call the tensor to be a nonsingular tensor. A refining tensor is a nonsingular tensor $\xi$ such that $\xi\left\langle\mathbb{R}^{\star}, \mathbb{R}^{\star}\right\rangle \subseteq \mathbb{R}^{\star}$. By $\mathbb{M}$ (resp. $\mathbb{T}$ ) we denote the set of refining Möbius maps (resp. refining tensors).

Definition 4.1 (Digit Set). A digit set is a finite set $\Phi$ of refining Möbius maps such that there is a total surjective map (a representation) $\rho$ from $\Phi^{\omega}$ to $\mathbb{R}^{\star}$ and that for all $f_{0} f_{1} \cdots \in \Phi^{\omega}$ we have

$$
\left\{\rho\left(f_{0} f_{1} \cdots\right)\right\}=\bigcap_{i=0}^{\infty} f_{0} \circ \cdots \circ f_{i}\left(\mathbb{R}^{\star}\right)
$$

Let $\Phi$ be a digit set. Let $\varphi \in \Phi$. For each $\mu \in \mathbb{M}$ (resp. $\xi \in \mathbb{T}$ ) we define the emission condition as the predicate:

$$
\begin{aligned}
\operatorname{Incl}(\mu, \varphi) & :=\mu\left(\mathbb{R}^{+}\right) \subseteq \varphi\left(\mathbb{R}^{+}\right), \\
\operatorname{Incl}(\xi, \varphi) & :=\xi\left\langle\mathbb{R}^{+}, \mathbb{R}^{+}\right\rangle \subseteq \varphi\left(\mathbb{R}^{+}\right) .
\end{aligned}
$$

First we show how we compose a Möbius map with a tensor. The idea is to replace $\xi\langle x, y\rangle$ for $x$ in $\mu(x)$. We use the ordinary "o" notation to denote composition:

$$
\left[\begin{array}{ll}
A & B \\
C & D
\end{array}\right] \circ\left[\begin{array}{llll}
a & b & c & d \\
e & f & g & h
\end{array}\right]=\left[\begin{array}{llll}
A a+B e & A b+B f & A c+B g & A d+B h \\
C a+D e & C b+D e & C c+D g & C d+D h
\end{array}\right] .
$$

\footnotetext{
${ }^{3}$ Instead of $\mathbb{R}^{\star}$, we could pick any proper closed subinterval of $\mathbb{R} \cup\{-\infty,+\infty\}$ [23, Chapter 5].
} 
Furthermore, we define the left product (denoted by $\bullet_{1}$ ) and the right product (denoted by $\bullet_{2}$ ) of a tensor and a Möbius map as follows:

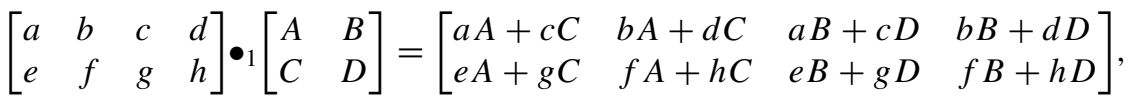

$$
\begin{aligned}
& {\left[\begin{array}{llll}
a & b & c & d \\
e & f & g & h
\end{array}\right] \cdot 2\left[\begin{array}{ll}
A & B \\
C & D
\end{array}\right]=\left[\begin{array}{llll}
a A+b C & a B+b D & c A+d C & c B+d D \\
e A+f C & e B+f D & g A+h C & g B+h D
\end{array}\right] .}
\end{aligned}
$$

Note that the above formulae are obtained by replacing $(A x+B) /(C x+D)$ (resp. $(A y+B) /(C y+D))$ for $x$ (resp. $y)$ in $\xi\langle x, y\rangle$.

Finally, we are able to present the NAlg in a functional form. This algorithm takes an expression tree in $\mathbb{E}(\mathbb{M}, \mathbb{T})$ to a stream of elements of $\Phi$ which represents a real number in $\mathbb{R}^{\star}$. The declaration is presented for two possible cases for the input: the case in which the root of the input expression tree is the unary node $\langle\langle\mu ; \theta\rangle\rangle$; and the case in which the root of the input expression tree is the binary node $\left\langle\left\langle\xi ; \theta_{1}, \theta_{2}\right\rangle\right\rangle$ :

$$
\begin{aligned}
& \mathrm{nf}_{\Phi}\left\langle\langle\mu ; \theta\rangle:= \begin{cases}\varphi: \mathrm{nf}_{\Phi}\left\langle\left\langle\varphi^{-1} \circ \mu ; \theta\right\rangle\right\rangle & \text { if } \exists \varphi \in \Phi, \quad \operatorname{Incl}(\mu, \varphi), \\
\operatorname{nf}_{\Phi}\left\langle\left\langle\mu \circ \operatorname{hd}\left(\mathrm{nf}_{\Phi}(\theta)\right) ; \mathrm{tl}\left(\mathrm{nf}_{\Phi}(\theta)\right)\right\rangle\right\rangle & \text { otherwise. }\end{cases} \right. \\
& \mathrm{nf}_{\Phi}\left\langle\left\langle\xi ; \theta_{1}, \theta_{2}\right\rangle\right\rangle:=\left\{\begin{array}{ccc}
\varphi: \mathrm{nf}_{\Phi}\left\langle\left\langle\varphi^{-1} \circ \xi ; \theta_{1}, \theta_{2}\right\rangle\right\rangle & \text { if } \quad \exists \varphi \in \Phi, & \operatorname{Incl}(\xi, \varphi), \\
\mathrm{nf}_{\Phi}\left\langle\xi \bullet_{1} \operatorname{hd}\left(\mathrm{nf}_{\Phi}\left(\theta_{1}\right)\right) \bullet_{2} \operatorname{hd}\left(\left(\mathrm{nf}_{\Phi}\left(\theta_{2}\right)\right)\right) ;\right. & \\
\left.\left.\mathrm{tl}\left(\mathrm{nf}_{\Phi}\left(\theta_{1}\right)\right), \mathrm{tl}\left(\mathrm{nf}_{\Phi}\left(\theta_{2}\right)\right)\right\rangle\right\rangle & \text { otherwise. }
\end{array}\right.
\end{aligned}
$$

The branch of the algorithm that deals with verifying the emission condition is called the emission step and the nested branch is called the absorption step.

Note that we assumed a digit set to be finite. Thus assuming $|\Phi|=n$ the condition $\exists \varphi \in \Phi$, Incl(_, $\left.\varphi_{i}\right)$ is a shorthand for $n$ conditions of the form $\left.\operatorname{Incl}_{(-}, \mu_{i}\right)$ for $0 \leq i<n$. That is to say, assuming that we order (arbitrarily) the elements of $\Phi$, the precise form of the above declaration should be written containing $n$ absorption steps, one for each digit.

The above definition is meaningful as it can be written as such in a functional programming language with lazy evaluation (e.g., Haskell). However, the functions in the type theory should be introduced using the introduction rules of the type theory. In this case it means that NAlg — being a map into the coinductive type of streams-should be expressed in term of the $\nu \mathrm{F}_{\mathrm{A}}$-INTRODUCTION rule. Therefore the above definition is not a valid type theoretic definition simply because it is not given in terms of the introduction rule.

In order to be able to formalise NAlg in our type theory we first list the type theoretic issues that we should tackle.

First, there is a type checking problem in the above presentation of NAlg. According to the type of input and output, the type of NAlg should be $\mathbb{E} \rightarrow \Phi^{\omega}$. This means that we should define the matrix multiplication (and left and right products) between a digit (which is a refining Möbius map) and a refining Möbius map which is a unary node of an expression tree. Note that in MTT there is no subtyping by inheritance. Hence we should use coercive subtyping, i.e., we should define a map between $\Phi$ and $\mathbb{M}$ and declare it to be a coercion [29]. This also solves the problem that in the absorption steps the argument of NAlg is an expression tree with streams as children. 
The second problem with formalising the above presentation of NAlg is more difficult to tackle and corresponds to the productivity of NAlg, which we discuss in the next section.

We conclude this section by pointing out how NAlg can be used to compute elementary function. In [26] expression trees of the following form are used to denote elementary real functions:

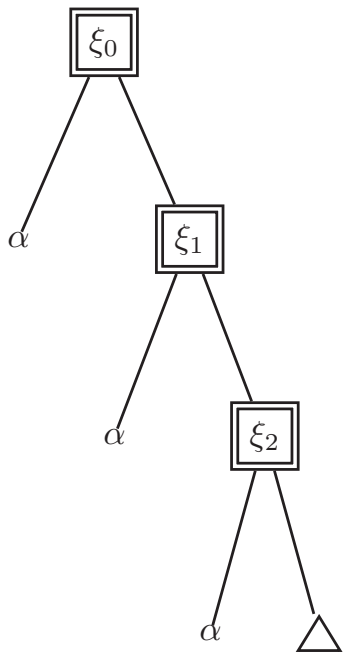

Here $\alpha$ is a stream which is the representation of a real number $x$ by elements of $\Phi$, i.e., $x=\rho\left(\varphi_{0} \varphi_{1} \cdots\right)$ for $\varphi_{i} \in \Phi$. The above expression tree is interpreted as

$$
\bigcap_{i=0}^{\infty} \xi_{0}\left\langle x, \xi_{1}\left\langle\cdots \xi_{i}\left\langle x, \mathbb{R}^{\star}\right\rangle\right\rangle .\right.
$$

If the $\xi_{i}$ 's are refining, then the above intersection is an intersection of a nested sequence of shrinking intervals. If this latter intersection is a singleton $\{y\}$ then $y=$ $\xi_{0}\left\langle x, \xi_{1}\left\langle x, \xi_{2}\langle x, \cdots\rangle\right\rangle\right\rangle$. Thus we see that such an expression tree can be considered as a real function that maps $x$ to $y$.

\section{Productivity}

If we formalise NAlg in our type theory, we should only use the rules of MTT plus those presented in Section 2. More specifically, the rule $v$-INTRODUCTION should be used to define infinite objects. This is the rule which builds the stream that is the outcome of NAlg. This rule is based on the coiteration scheme and hence it can only be used to define infinite objects whose construction does not involve an unbounded search. This also would have been the case had we chosen more complex schemes of corecursion or the dual of course-of-value recursion to propose an alternative introduction rule. However, one can see that the absorption steps of NAlg require an unbounded search: the algorithm absorbs from the input expression tree and modifies the input until the condition $\operatorname{Incl}(-,-)$ holds.

This means that NAlg might not produce output for some inputs, i.e., it might be a partial function. If a function on infinite objects produces an output for a given in- 
put, we say that this function is productive for that input. Productivity is dual to the notion of termination which is used to ensure the totality of recursive functions. The subtlety in the definition of productivity is that the output is the entirety of the infinite object and not just finite segments of it, i.e., productive means provably infinite. That means that for a function to be productive we should have a modulus of convergence that ensures the output will have arbitrary large initial segments. Such a modulus of convergence is a function from $\mathbb{N}$ to $\mathbb{N}$ that specifies for each $n$ how much of the input should be processed to output $n$ elements. This in turn means that the complexity of the function should be known. This informal argument can be made more formal when expressed in the language of the theory of dcpo's. Therefore we impose an order structure on the set of streams and the set of expression trees that will capture partiality and undefinedness.

First we present a formal definition of productivity using the theory of partially ordered sets. A subset $A$ of a poset is directed if it is nonempty and each pair of elements of $A$ has an upper bound in $A$. Recall that a $d c p o$ - directed-complete partial order-is a poset in which every directed subset has a supremum. A pointed dcpo is a dcpo lifted by adding the bottom element $\perp$. A maximal element of a dcpo is an element for which there is no element strictly above it. A continuous function between two dcpo's is a monotone function that preserves suprema of the directed subsets.

Definition 5.1. We define $[\mathrm{A}]$ to be the pointed dcpo of partial lists and streams over set $A$ as follows. The elements of $[\mathrm{A}]$ are partial lists and partial streams. A partial list is a finite list of elements of $A \cup\left\{\perp_{A}\right\}$, where $\perp_{A}$ is a fresh element added to $A$ and a partial stream is an element of $\left(A \cup\left\{\perp_{A}\right\}\right)^{\omega}$. In order to define the order on [A] let $\sqsubseteq_{A}$ be the order on the flat-domain $A \cup\left\{\perp_{A}\right\}$ (i.e., $\forall a \in A, \perp_{A} \sqsubseteq a$ ). The order on [A] can be defined as

$$
v_{1} \lesssim v_{2}:= \begin{cases}\forall 0 \leq i<\operatorname{length}\left(v_{1}\right), \quad v_{1}(i) \sqsubseteq_{A} v_{2}(i) & \text { if } v_{1} \text { is a partial list; } \\ \forall i \geq 0, \quad v_{1}(i) \sqsubseteq_{A} v_{2}(i) & \text { if } v_{1} \text { is a stream. }\end{cases}
$$

Here $v(i)$ denotes the $i$ th element of $v$ (the counting starts from 0, e.g., $v(0)=\mathrm{hd}(v)$ ).

The above dcpo provides semantics for reduction (i.e., evaluation or unfolding) of declarations defining a stream or a function on streams in, e.g., Haskell. Partial lists are finite objects and denote finite evaluations, while partial streams denote infinite evaluations. As an example the empty partial list $\perp_{[\mathbb{N}]}$ refers to an unevaluated (possibly nonproductive) stream of natural numbers. On that account, the one-element partial list $0: \perp_{[\mathbb{N}]}$ denotes a one-step reduction of a declaration that has output 0 immediately after one reduction; while $\perp_{\mathbb{N}}: \perp_{[\mathbb{N}]}$ denotes a one-step reduction of a declaration that has not output anything (equivalently has output $\perp_{\mathbb{N}}$ ). Finally, the infinite stream $\left(\perp_{\mathbb{N}}\right)^{\omega}$ denotes a declaration that never outputs anything after an infinite number of reduction steps, e.g., the declaration that filters out the tautology $x=x$ from a stream of natural numbers. Thus each partial object in the above dcpo denotes partial evaluation of a declaration in Haskell and hence the depo does not include more partial objects than necessary. 
Definition 5.2. We define $[A ; B]$ to be the pointed dcpo of partial expression trees with $A$-elements and $B$-operations as follows. The elements of $[A ; B]$ are finite and infinite trees in which every node has one or two children. The unary nodes contain elements of $A \cup\left\{\perp_{A}\right\}$ and the binary nodes contain the elements of $B \cup\left\{\perp_{B}\right\}$. To define the order note that each branch of a partial expression tree is a partial list or stream. Hence we say $\theta_{1} \unlhd \theta_{2}$ if and only if for all $\sigma_{1}$ if $\sigma_{1}$ is a branch of $\theta_{1}$ then there is a branch $\sigma_{2}$ of $\theta_{2}$ such that $\sigma_{1} \lesssim \sigma_{2}$.

Note that the above elucidation for $[\mathrm{A}]$ is valid for $[A ; B]$ as well: each partial expression tree denotes partial evaluation of a Haskell declarations for expression trees or functions on expression trees. In particular, partial finite trees denote the finite step reductions.

It is easily proved that $\langle[A], \lesssim\rangle$ and $\langle[A ; B], \unlhd\rangle$ are pointed dcpo's [23, Lemmas 4.5.2 and 4.5.5]. At this point we can define the productivity as a property of continuous functions into [A] (which is all we need in case of NAlg).

Definition 5.3 (Productive, Terminative). For nonempty sets $A$ and $B$, in the dcpo's $[\mathrm{A}]$ and $[A ; B]$ we shall call each maximal element a productive element.

For finite partial lists we use the dual term terminative. A finite partial list $\sigma$ in $[A]$ is terminative if and only if,

$$
\forall 0 \leq i<\text { length }(\sigma), \quad \sigma(i) \text { is maximal. }
$$

Note that the empty partial list is vacuously terminative.

We call a continuous function between two pointed dcpo's productive if it takes productive elements to productive elements.

It is immediate from this definition that a maximal element of the function space of standard dcpo's is productive. On the other hand, in the function space, productivity is a weaker notion than maximality [23, Example 4.6.3]. Furthermore, it is immediate from the above definition that composition of two productive functions is productive.

It is not always easy to verify the productivity of continuous functions directly. For the case of productive streams there is a criterion which is usually easier to apply than the direct application of the above definition [30, Theorem 32]. Our goal is to present a similar criterion for continuous functions that output streams; such a criteria can then be applied to NAlg. As we are mainly interested in productivity of fixpoints, we will use the following equation which holds in every pointed dcpo [1] ( $\mu f$ denotes the fixpoint of continuous function $f$ ):

$$
\mu f=\bigsqcup_{n \in \mathbb{N}}^{\uparrow} f^{n}(\perp) .
$$

In what follows we assume $D$ to be a pointed depo with $F$ a continuous functional from $D \longrightarrow[A]$ to $D \longrightarrow[A]$. We denote $F^{j}\left(\perp_{D \longrightarrow[A]}\right)$ by $f^{j}$ which is a function from $D$ to $[A]$. Note that because of the monotonicity of $F$ if $i \leq j$ then $f^{i} \sqsubseteq f^{j}$. By $(\alpha)_{j}$ we 
denote the list consisting of the first $j$ elements of $\alpha$. We introduce a predicate which we use as a criterion for the productivity of fixpoints.

Definition 5.4. For $d \in D$ we define the predicate $\operatorname{cont}(F, d)$ as

$$
\operatorname{cont}(F, d):=\forall j \geq 0, \exists k \geq 0, \quad\left(f^{k}(d)\right)_{j} \text { is terminative. }
$$

If $\operatorname{cont}(F, d)$, then we say $F$ is terminative at $d$. We define the predicate $\operatorname{Cont}(F)$ as the global version of cont on all maximal elements, i.e.,

$\operatorname{Cont}(F):=(\forall d \in D, d$ is maximal $\Longrightarrow \operatorname{cont}(F, d))$.

If $\operatorname{Cont}(F)$ holds, then we say that $F$ is terminative.

Proposition 5.5. If $F$ is terminative then $\mu F$ is productive.

Proof. This proposition is derived as Corollary 4.6 .8 in [23].

The converse of the above proposition does not hold in general. However, the converse result holds if we restrict ourselves to dcpo's in which the maximal elements are accessible [23, Lemma 4.6.10]. (We call an element $d$ of a pointed dcpo accessible, if there are finitely many elements strictly between $d$ and $\perp$.)

\section{Productivity of NAlg}

For our type theoretic implementation of NAlg we need to apply Proposition 5.5 and extract a modulus of convergence from the proof of terminativity. For this, it is enough to have a pointwise proof of productivity. However, in this section (slightly diverging from our type theoretic motives) we discuss the productivity of NAlg on some families of expression trees. This relates to the way Edalat and Potts use NAlg to interpret real functions as families of expression trees. Clearly this will result in the modulus of convergence that we need for pointwise productivity and we follow on with that in the next section.

We presented $\mathrm{nf}_{\Phi}$ for a fixed representation that is given by the digit set $\Phi$. Therefore it is natural to expect that the productivity behaviour of NAlg depends on $\Phi$. This is because each emission step of NAlg emits an output when the emission conditiona topological property of the chosen digit set-holds. However, bearing in mind that NAlg is used for evaluating real functions the value of its output should be independent of the representation we choose. This apparent discrepancy can be explained by the fact that for any digit set $\Phi$ if $\Phi$, as a representation, has enough redundancy then $\mathrm{nf}_{\Phi}$ outputs a representation of the same real number.

We base our work on admissible representations, a notion borrowed from type two theory of effectivity [31]. 
Definition 6.1 (Admissible Representation for $\mathbb{R}^{\star}$ ). A map $p: \Phi^{\omega} \longrightarrow \mathbb{R}^{\star}$ is an admissible representation of $\mathbb{R}^{\star}$ if the following conditions hold:

(i) $p$ is continuous with respect to the product topology of the discrete topology on $\Phi$;

(ii) $p$ is surjective;

(iii) $p$ is maximal, i.e., for every (partial) continuous $r: \Phi^{\omega} \longrightarrow \mathbb{R}^{\star}$, there is a continuous $f: \Phi^{\omega} \longrightarrow \Phi^{\omega}$ such that $r=p \circ f$.

It is known that such representations for real numbers have enough redundancy to be used for computing real computable functions such as elementary functions [31]. Therefore we restrict ourselves to digit sets that lead to admissible representations. Note that the definition of admissibility is given for arbitrary representations, while we are working with representations based on Möbius maps. Here we give a criterion for digit sets that are admissible and simplifies the above definition.

Let $\mathbf{S}(x)=(x-1) /(x+1)$ and, given an interval $[x, y] \subseteq \mathbb{R}^{\star}$, let $\operatorname{diam}([x, y])=$ $|\mathbf{S}(x)-\mathbf{S}(y)|$. Note that Sis a bijection between $\mathbb{R}^{\star}$ and $[-1,1]$ and diam is a metric on the $\mathbb{R}^{\star}$ (which is one-point compactification of $[0,+\infty)$ ) for capturing the diameter of possibly infinite intervals. Using this we define the following.

Definition 6.2 (Admissible Digit Set). Let $\Phi$ be a finite set of refining increasing Möbius maps. We call $\Phi$ an admissible digit set for $\mathbb{R}^{\star}$ if both following conditions hold:

(a) $\lim _{j \rightarrow \infty} \max \left\{\operatorname{diam}\left(\varphi_{0} \circ \varphi_{1} \circ \cdots \circ \varphi_{j-1}\left(\mathbb{R}^{\star}\right)\right) \mid \varphi_{0}, \ldots, \varphi_{j-1} \in \Phi\right\}=0$;

(b) $\bigcup_{\varphi_{i} \in \Phi} \varphi_{i}\left(\mathbb{R}^{+}\right)=\mathbb{R}^{+}$.

The above condition 6.2(a) ensures that each infinite composition of elements of $\Phi$ is a singleton. Condition 6.2(b) requires the range of the digits (considered as Möbius maps) to be a finite covering of $\mathbb{R}^{+}$by intervals with overlapping interiors.

It is immediate that an admissible digit set is a digit set. Therefore there exists a map $\operatorname{Rep}_{\Phi}: \Phi^{\omega} \longrightarrow \mathbb{R}^{\star}$ such that $\bigcap_{i=0}^{\infty} \varphi_{0} \circ \cdots \circ \varphi_{i}\left(\mathbb{R}^{\star}\right)=\left\{\operatorname{Rep}_{\Phi}\left(\varphi_{0} \varphi_{1} \cdots\right)\right\}$. The following proposition justifies the above definition. The proof can be found in Theorem 5.4.9 of [23].

Proposition 6.3. Let $\Phi$ be an admissible digit set. Then $\mathbf{R e p}_{\Phi}$ is an admissible representation.

As an example, if $\varphi_{\mathbf{L}}:=x /(x+1)$ and $\varphi_{\mathbf{R}}:=x+1$ then $\Phi_{2}=\left\{\varphi_{\mathbf{L}}, \varphi_{\mathbf{R}}\right\}$ is a digit set which is not admissible. However, extending it with $\varphi_{\mathbf{N}}(x):=(2 x+1) /(x+2)$ we obtain $\Phi_{3}=\left\{\varphi_{\mathbf{L}}, \varphi_{\mathbf{R}}, \varphi_{\mathbf{N}}\right\}$, which is an admissible digit set [23, Section 5.7].

The next thing we need is a basic quantity which characterises the redundancy of an admissible digit set. 
Definition 6.4. Let $\Phi$ be an admissible digit set. We define the redundancy of $\Phi$ as

$$
\operatorname{red}(\Phi)=\min \left\{\left|\mathbf{S}\left(\varphi_{i}(0)\right)-\mathbf{S}\left(\varphi_{j}(+\infty)\right)\right| \mid \varphi_{i}, \varphi_{j} \in \Phi, \varphi_{i}(0) \neq \varphi_{j}(+\infty)\right\}
$$

The above definition is based on the intuitive idea that the more overlap between ranges of the digits, the more choices one has for representing real numbers. The intended meaning is that for two admissible digit sets $\Phi_{1}$ and $\Phi_{2}$, with $\left|\Phi_{1}\right|=\left|\Phi_{2}\right|$, if $\operatorname{red}\left(\Phi_{1}\right)>\operatorname{red}\left(\Phi_{2}\right)$ then $\Phi_{1}$ has more redundancy. Note that this intended meaning does not work for adding extra digits (which decreases red) but rather for comparing the redundancy of two digits sets with the same number of digits.

From now on we work with an admissible digit set $\Phi$. Using the following important property of redundancy we can show that the emission condition holds after finitely many absorption steps.

Proposition 6.5. Let $\mu \in \mathbb{M}$ be such that $\operatorname{diam}\left(\mu\left(\mathbb{R}^{\star}\right)\right)<\operatorname{red}(\Phi)$. Then there exists $\varphi_{i} \in \Phi$ such that $\operatorname{Incl}\left(\mu, \varphi_{i}\right)$.

Proof. Let $k=|\Phi|$. By Condition 6.2(b), we know that $\mu\left(\mathbb{R}^{+}\right) \subseteq \bigcup_{i=0}^{k-1} \varphi_{i}\left(\mathbb{R}^{+}\right)$where $\mu\left(\mathbb{R}^{+}\right)$and all $\varphi_{i}\left(\mathbb{R}^{+}\right)$'s are intervals. Hence, either there is an $i$ such that $\varphi_{i}(0) \in \mu\left(\mathbb{R}^{+}\right)$ or there is an $i$ such that $\mu\left(\mathbb{R}^{+}\right) \subseteq \varphi_{i}\left(\mathbb{R}^{+}\right)$. In the latter case, we have $\operatorname{Incl}\left(\mu, \varphi_{i}\right)$.

For the former case, suppose there is an $x \in \mu\left(\mathbb{R}^{+}\right)$such that $x=\varphi_{i}(0)$ for some $\varphi_{i} \in \Phi$. Since $\Phi$ is finite without loss of generality we can assume that $x$ is the smallest number with this property, i.e.,

$$
\forall i^{\prime}<k, \quad \varphi_{i^{\prime}}(0)<\varphi_{i}(0) \Rightarrow \mathbf{S}\left(\varphi_{i^{\prime}}(0)\right)<\mathbf{S}(\mu(0)) .
$$

By assumption, $\operatorname{diam}\left(\mu\left(\mathbb{R}^{\star}\right)\right)<\operatorname{red}(\Phi)$ and so

$$
\mathbf{S}(\mu(+\infty))-\mathbf{S}(\mu(0))<\operatorname{red}(\Phi)
$$

It follows that

$$
\mathbf{S}(\mu(+\infty))-\mathbf{S}(x)<\operatorname{red}(\Phi)
$$

so

$$
\mathbf{S}\left(\mu\left(\mathbb{R}^{+}\right)\right) \varsubsetneqq[\mathbf{S}(\mu(0)), \mathbf{S}(x)+\operatorname{red}(\Phi)] .
$$

Since $x \in \mathbb{R}^{+}$, there is a $\varphi_{j} \in \Phi$ with $x \in \varphi_{j}\left(\mathbb{R}^{+}\right)$. Note that $\varphi_{j}(0)<\varphi_{i}(0)$ and hence by (6.1) $\mathbf{S}\left(\varphi_{j}(0)\right)<\mathbf{S}(\mu(0))$. Moreover, the minimality of $\operatorname{red}(\Phi)$ in Definition 6.4 means that the endpoint $\mathbf{S} \circ \varphi_{j}(+\infty)$ is at least at a distance $\operatorname{red}(\Phi)$ from $\mathbf{S}(x)$. In other words,

$$
[\mathbf{S}(\mu(0)), \mathbf{S}(x)+\operatorname{red}(\Phi)] \varsubsetneqq\left[\mathbf{S}\left(\varphi_{j}(0)\right), \mathbf{S}\left(\varphi_{j}(+\infty)\right)\right]
$$

From this and (6.2) it follows that $\operatorname{Incl}\left(\mu, \varphi_{j}\right)$ holds. 
To state a similar result for the tensors note that if $I_{1}, I_{2}$ are two closed intervals and $\xi \in \mathbb{T}$, then $\xi\left\langle I_{1}, I_{2}\right\rangle$ is a closed interval. The proof of the following result is similar to the above proof.

Proposition 6.6. Let $\xi \in \mathbb{T}$ such that $\operatorname{diam}\left(\xi\left\langle\mathbb{R}^{\star}, \mathbb{R}^{\star}\right\rangle\right)<\operatorname{red}(\Phi)$. Then there exists $\varphi_{i} \in \Phi$ such that $\operatorname{Incl}\left(\xi, \varphi_{i}\right)$.

Propositions 6.5 and 6.6 are the main tools for proving productivity of NAlg. They give us enough information about the complexity of NAlg in the sense that at each step they will give us the pointwise delay, i.e., the local upper bound for the number of steps that should be waited before the next element of output is emitted.

As can be seen, at each step of the evaluating $\mathrm{nf}_{\Phi}$, this bound is dependent on two parameters: (1) the redundancy red $(\Phi)$, and (2) the length of the interval $\operatorname{diam}\left(\mu\left(\mathbb{R}^{\star}\right)\right)$ $\left(\operatorname{or} \operatorname{diam}\left(\xi\left\langle\mathbb{R}^{\star}, \mathbb{R}^{\star}\right\rangle\right)\right)$. Clearly (1) is a topological property of the digit set and (2) is a combination of a metric property of the matrix (or tensor) that is the root of the input expression tree to $\mathrm{nf}_{\Phi}$ together with the convergency rate of the input stream of digits. Of these two parameters, the first one is constant during the evaluation of the algorithm; however, the second one is a dynamic property that changes with each new recursive call to $\mathrm{nf}_{\Phi}$ with a new input expression tree. The recursive calls in the emission branches modify the head by multiplying it with the inverse of the emitted digit while recursive calls in the absorption step ask for more digits from the subtree in a nested way. Therefore if we can find an upper bound for all the nested local upper bounds in absorption steps, it will lead us to a modulus of convergence that can be used in the proof of the terminativity and subsequently the productivity of NAlg.

Finding such upper bound is easy if the structure of the initial input is simple. The "simplest" possible expression tree in this sense is a stream of elements of $\Phi$ (recall that $\Phi \subset \mathbb{M})$. In this case $\mathrm{nf}_{\Phi}$ will act as the identity function on real numbers, i.e., it evaluates to another stream of $\Phi$ that is a representation for the same real number as the original stream.

The next level up from the identity function is the evaluation of $\mathrm{nf}_{\Phi}$ on the expression tree containing a Möbius map $\mu \notin \Phi$ prepended to a stream $\alpha \in \Phi^{\omega}$. In this case NAlg will evaluate a representation for $\mu(x)$ where $\operatorname{Rep}_{\Phi}(\alpha)=x$. This simple case of NAlg is known as the homographic algorithm. In this case finding a modulus of convergence is easy because the subsequent calls to $\mathrm{nf}_{\Phi}$ in the absorption steps are not nested, and thus the upper bound needed for point (2) above is estimable. In Section 5.6 of [23] this idea is used to prove the productivity of the homographic algorithm. We will return to the exact statement of this theorem shortly, after we introduce some notation.

Definition 6.7 (Open Expression Tree). Let $\theta \in[\mathbb{M} ; \mathbb{T}]$. Then $\theta$ is open if, for every $j>0$, the initial segment of length $j$ of each branch of $\theta$ is a terminative list.

Let $\alpha \in \Phi^{\omega}$. For an open expression tree $\theta$ we define $\theta+\alpha$ to be the productive expression tree which is obtained by concatenating finite branches of $\theta$ with $\alpha$.

As an example suppose $\theta^{\prime}$ is a productive expression tree and let $\theta$ be the following partial expression tree (left). Then its padding by $\Phi$-normal form $\alpha$ is illustrated (right) 
(here $\triangle$ denotes another expression tree which is attached to the depicted expression tree) as follows:
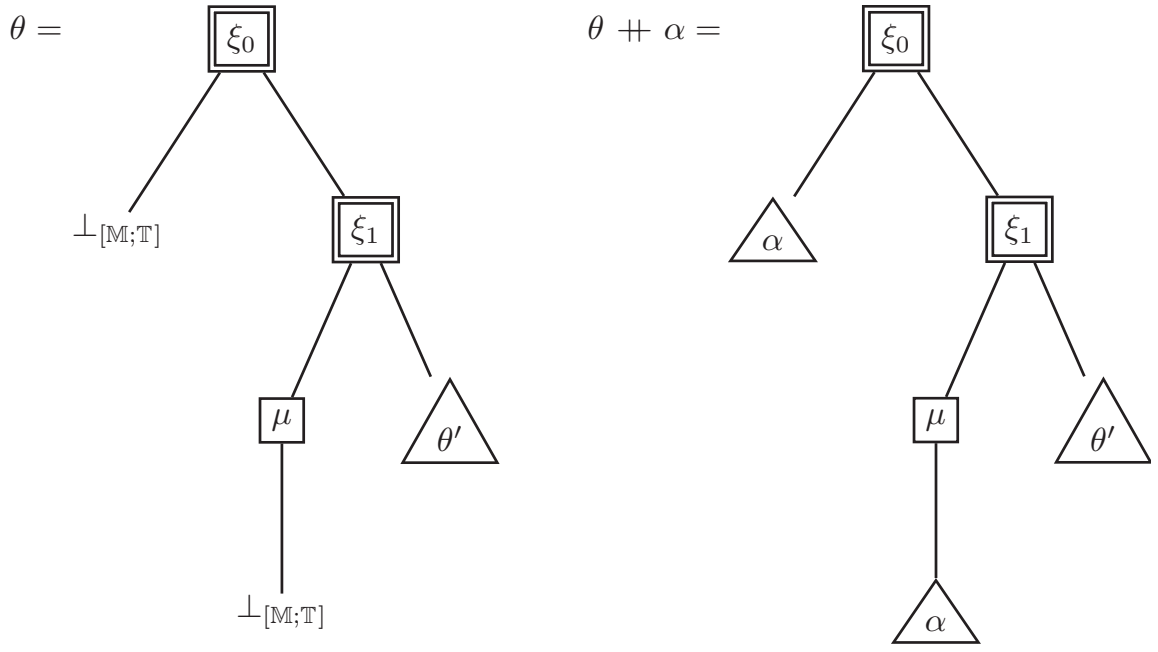

Note that the above definition is analogous to viewing finite lists as initial segments of streams. According to the order on $[\mathbb{M} ; \mathbb{T}]$, having $\perp_{[\mathbb{M} ; \mathbb{T}]}$ at the leaves of finite branches of an open expression tree $\theta$ entails that $\theta \unlhd \theta+\alpha$ for all $\alpha \in \Phi^{\omega}$. Thus open expression trees can be considered as minimal elements in special families of expression trees which we define below. This is in accordance with viewing $\perp$ in a dcpo as the object containing the least amount of information.

Definition 6.8. Let $\dot{\theta}$ be an open expression tree. Then we define the family of expression trees specified by $\dot{\theta}$ to be the set $\Theta(\dot{\theta})=\left\{\dot{\theta}+\alpha \mid \alpha \in \Phi^{\omega}\right\}$.

Using the above notation, we can describe the homographic algorithm as the restriction of NAlg on $\left.\Theta\left(\left\langle\mu ; \perp_{[\mathbb{M} ; \mathbb{T}]}\right\rangle\right)\right)$. Similarly we have the quadratic algorithm, which is the restriction of NAlg on $\Theta\left(\left\langle\left\langle\xi ; \perp_{[\mathbb{M} ; \mathbb{T}]}, \perp_{[\mathbb{M} ; \mathbb{T}]}\right\rangle\right)\right.$.

Proposition 6.9. The homographic algorithm is productive.

Proof. This proposition is derived as Corollary 5.6.1 in [23].

The proof of the above theorem uses Proposition 6.5 and the fact that for $\mu=\left[\begin{array}{ll}a & b \\ c & d\end{array}\right]$ we have (see [18])

$$
\operatorname{diam}(\mu,[x, y])=\operatorname{diam}(x, y) \cdot|\operatorname{det} \mu| \cdot \frac{(x+1)(y+1)}{((a+c) x+b+d)((a+c) y+b+d)} .
$$

To prove the productivity of the quadratic algorithm we need a similar result about the shrinking effect of refining tensors on intervals. First we need to define the diameter 
for application of the refining quadratic maps. Let $\xi \in \mathbb{T}$. Since $\xi$ is nonsingular and refining, it is directionally monotone on the square $\mathbb{R}^{\star} \times \mathbb{R}^{\star}$, i.e., it is monotone in each variable by fixing the other variable. Therefore the value of $\xi\left\langle\mathbb{R}^{\star}, \mathbb{R}^{\star}\right\rangle$ assumes its extrema on the corners of this square. This fact is trivially inherited by subsquares of $\mathbb{R}^{\star} \times \mathbb{R}^{\star}$; we state this as a lemma.

Proposition 6.10. Let $I_{1}=\left[\underline{l}_{1}, \bar{l}_{1}\right], I_{2}=\left[\underline{l}_{2}, \bar{l}_{2}\right] \subseteq \mathbb{R}^{\star}$ and let $\xi \in \mathbb{T}$. Then $\xi\left\langle I_{1}, I_{2}\right\rangle=$ $\left[\underline{l}_{3}, \bar{l}_{3}\right]$, where

$$
\begin{aligned}
& \underline{l}_{3}=\min \left\{\xi\left\langle\underline{l}_{1}, \bar{l}_{1}\right\rangle, \xi\left\langle\underline{l}_{1}, \bar{\imath}_{2}\right\rangle, \xi\left\langle\underline{l}_{2}, \bar{\imath}_{1}\right\rangle, \xi\left\langle\underline{l}_{2}, \bar{\imath}_{2}\right\rangle\right\}, \\
& \bar{l}_{3}=\max \left\{\xi\left\langle\underline{l}_{1}, \bar{\imath}_{1}\right\rangle, \xi\left\langle\underline{l}_{1}, \bar{\imath}_{2}\right\rangle, \xi\left\langle\underline{l}_{2}, \bar{l}_{1}\right\rangle, \xi\left\langle\underline{l}_{2}, \bar{l}_{2}\right\rangle\right\} .
\end{aligned}
$$

Note that the $\underline{l}_{i} \mathrm{~s}$ and $\bar{l}_{i} \mathrm{~s}$ are rational numbers, and hence all comparisons are decidable.

Since the value of the diameter for the quadratic maps is determined by a max operator, it is difficult to find a uniform closed formula for this value. Even for particular digit sets, such as $b$-ary admissible digit sets, the closed form is difficult to find and one has to work with upper and lower bounds [11], [18]. However, as far as the analysis of the normalisation algorithm is concerned, the following result is enough.

Proposition 6.11. Let $\xi \in \mathbb{T}$. Then for every $\varepsilon>0$ there exists $\vartheta_{1}, \vartheta_{2}>0$ such that

$$
\forall I_{1}, I_{2} \subseteq \mathbb{R}^{\star}, \quad \operatorname{diam}\left(I_{1}\right)<\vartheta_{1} \wedge \operatorname{diam}\left(I_{2}\right)<\vartheta_{2} \Longrightarrow \operatorname{diam}\left(\xi\left\langle I_{1}, I_{2}\right\rangle\right)<\varepsilon
$$

Proof. Note that $\xi$ is refining and therefore its denominator does not vanish on $\mathbb{R}^{\star} \times \mathbb{R}^{\star}$. The result then is a straightforward consequence of the continuity of $\xi$.

Here we sketch the main point of the proof of the productivity, skipping the detailed yet straightforward steps of creating a higher-order functional and proving its terminativity. That is, we prove that the quadratic algorithm outputs arbitrary long lists of digits. A rigorous way of proving the terminativity (in the sense of increasing the length of the output) is done in [23] for the homographic algorithm, and for the quadratic algorithm it is equally tedious and straightforward.

Proposition 6.12. The quadratic algorithm is productive.

Proof. We should prove that given a $\xi$, for any $n$, $\mathrm{nf}_{\Phi}$ restricted to $\left.\Theta\left(\left\langle\xi ; \perp_{[\mathbb{M} ; \mathbb{T}]}, \perp_{[\mathbb{M} ; \mathbb{T}]}\right\rangle\right)\right)$ outputs $n$ digits. That is, we have to prove that given two streams $\alpha_{1}, \alpha_{2} \in \Phi^{\omega}, \mathrm{nf}_{\Phi}\left\langle\left\langle\xi ; \alpha_{1}, \alpha_{2}\right\rangle\right\rangle$ outputs $n$ digits.

We prove by induction on $n$ with the base case being $n=1$. First apply Proposition 6.11 to obtain $\vartheta_{1}$ and $\vartheta_{2}$ such that for all $I_{1}, I_{2} \subseteq \mathbb{R}^{\star}$,

$\operatorname{diam}\left(I_{1}\right)<\vartheta_{1} \wedge \operatorname{diam}\left(I_{2}\right)<\vartheta_{2} \Longrightarrow \operatorname{diam}\left(\xi\left\langle I_{1}, I_{2}\right\rangle\right)<\operatorname{red}(\Phi)$. 
Now, applying property 6.2(a) in the definition of an admissible digit set, choose $N>0$ such that

$$
\max \left\{\operatorname{diam}\left(\varphi_{0} \circ \varphi_{1} \circ \cdots \circ \varphi_{N-1}\left(\mathbb{R}^{\star}\right)\right) \mid \varphi_{0}, \ldots, \varphi_{N-1} \in \Phi\right\}<\min \left\{\vartheta_{1}, \vartheta_{2}\right\} .
$$

Take Möbius maps $\zeta_{1}$ and $\zeta_{2}$ such that

$$
\zeta_{1}=\left(\mathrm{nf}_{\Phi}\left(\alpha_{1}\right)\right)_{N}=\left(\alpha_{1}\right)_{N}, \quad \zeta_{2}=\left(\mathrm{nf}_{\Phi}\left(\alpha_{2}\right)\right)_{N}=\left(\alpha_{2}\right)_{N}
$$

i.e., $\zeta_{1}\left(\right.$ resp. $\left.\zeta_{2}\right)$ is a finite composition of the first $N$ element of $\alpha_{1}$ (resp. $\left.\alpha_{2}\right)$. Therefore, by (6.4) it follows that

$$
\operatorname{diam}\left(\zeta_{1}\left(\mathbb{R}^{\star}\right)\right)<\vartheta_{1}, \quad \operatorname{diam}\left(\zeta_{2}\left(\mathbb{R}^{\star}\right)\right)<\vartheta_{2},
$$

whence by taking $I_{1}=\zeta_{1}\left(\mathbb{R}^{\star}\right)$ and $I_{2}=\zeta_{2}\left(\mathbb{R}^{\star}\right)$ in (6.3) we conclude

$$
\begin{aligned}
\operatorname{diam}\left(\xi\left\langle\zeta_{1} \mathbb{R}^{\star}, \zeta_{2} \mathbb{R}^{\star}\right\rangle\right) & =\operatorname{diam}\left(\xi \bullet \bullet_{1} \zeta_{1} \bullet 2 \zeta_{2}\left\langle\mathbb{R}^{\star}, \mathbb{R}^{\star}\right\rangle\right) \\
& <\operatorname{red}(\Phi)
\end{aligned}
$$

Therefore by Proposition $6.6 \mathrm{nf}_{\Phi}\left\langle\left\langle\xi ; \alpha_{1}, \alpha_{2}\right\rangle\right\rangle$ outputs one digit.

For the induction step assume $\mathrm{nf}_{\Phi}\left\langle\left\langle\xi ; \alpha_{1}, \alpha_{2}\right\rangle\right\rangle$ has output $n$ digits. Note that in the quadratic algorithm after each recursion call in the emission or absorption steps the head node of the expression tree that is passed as the recursive argument is always a tensor. In the absorption step this is because $\bullet_{1}$ and $\bullet_{2}$ produce a new tensor by composing a tensor with two Möbius maps. In the emission step this is due to the fact that the composition of $\varphi^{-1}$ (a Möbius map) and a tensor is a tensor. Thus, let $\xi^{\prime}$ be the head of the new input expression tree after outputting the $n$th digit and let $\alpha^{\prime}{ }_{1}$ and $\alpha^{\prime}{ }_{2}$ be its children, i.e.,

$$
\mathrm{nf}_{\Phi}\left\langle\left\langle\xi ; \alpha_{1}, \alpha_{2}\right\rangle\right\rangle=\varphi_{i_{0}} \cdots \varphi_{i_{n}} \circ \mathrm{nf}_{\Phi}\left\langle\left\langle\xi^{\prime} ; \alpha_{1}^{\prime}, \alpha_{2}^{\prime}\right\rangle\right\rangle .
$$

Then we can repeat the reasoning that we did in the case $n=1$ for $\xi^{\prime}, \alpha^{\prime}{ }_{1}$ and $\alpha^{\prime}{ }_{2}$ and prove that $\left\langle\xi^{\prime} ; \alpha_{1}^{\prime}{ }_{1}, \alpha_{2}^{\prime}{ }_{2}\right\rangle$ produces one more element which will be the $(n+1)$ st element of the output for $\mathrm{nf}_{\Phi}\left\langle\left\langle\xi ; \alpha_{1}, \alpha_{2}\right\rangle\right\rangle$. This completes the proof by induction.

Following this line of thought we observe that if an expression tree contains only a finite number of tensors and a finite number of Möbius maps $\mu \notin \Phi$, then we expect to be able to prove the productivity by finding an upper bound for the delay on all possible nested recursive calls. The intuitive reason for this is that in such cases after finitely many nested calls in absorption steps we arrive at streams of elements of $\Phi$.

We state the next result in terms of accessible open expression trees which are open expression trees with finitely many tensors and Möbius maps. The proof uses the finitary character of the families specified by accessible open expression trees. In other words, we use structural induction on the set of accessible open expression trees. For a property $P$ on this set, the induction principle that we use can be stated as

$$
\frac{P(\perp) \quad P(\dot{\theta}) \rightarrow\langle\langle\mu ; \dot{\theta}\rangle\rangle \quad P\left(\dot{\theta}_{1}\right) \wedge\left(\dot{\theta}_{2}\right) \rightarrow\left\langle\left\langle\xi ; \dot{\theta}_{1}, \dot{\theta}_{2}\right\rangle\right.}{\forall \dot{\theta}, P(\dot{\theta})} .
$$


This induction principle is provable using Definition 6.7 and the definition of accessibility on dcpo's. ${ }^{4}$ Therefore we accept this induction principle.

Using this induction principle and by pattern matching on the input we can prove a statement about the productivity of NAlg on the family of accessible open expression trees. The proof, which uses Proposition 5.5, is very verbose and is sketched in Section 5.6.3 of [23]. The proof follows the same path as the proof for the simple case of a homographic algorithm which is given in detail in Lemma 5.6.10 of [23].

Proposition 6.13. Let $\Phi$ be an admissible digits set. Then, for any accessible open tree $\dot{\theta}$, NAlg is productive on $\Theta(\dot{\theta})$.

Being an accessible open expression tree is only a sufficient condition for productivity. In fact one can show that accessible open expression trees correspond to rational functions, i.e., a polynomial (in one variable) divided by another polynomial. ${ }^{5}$

There are more complex expression trees for which NAlg is know to be productive. Among them are the families corresponding to the elementary real functions. Such families have the property that they consist of a recursively generated infinite stream of tensors on one branch of the tree and streams of real numbers on the other branches. ${ }^{6}$ The proof of productivity in those cases is given in a per-case basis by analysing the convergence of the continued fraction [26], [18]. It is still an open problem to find a generic proof of productivity for such expression trees.

\section{Formalisation of the Semantical Method}

Proposition 6.13 ensures that when $\Phi$ is an admissible digits set and $\theta$ is an accessible open expression tree, $\mathrm{nf}_{\Phi}(\theta)$ should be formalisable in our type theory. The main point is that we have to prove the terminativity which is an existential proposition (a $\Sigma$-type). Since our proofs are constructive, for each accessible open expression tree $\theta$ one can extract a function $\varkappa_{\theta}: \mathbb{N} \longrightarrow \mathbb{N}$ that is the modulus of convergence of $\operatorname{nf}_{\Phi}(\theta)$. Then NAlg can be considered as a partial function modulo the existence of such a function. In this section we show how to formalise NAlg in this way. This approach is similar to the iterative approach of [2] for proving termination of nonstructurally recursive functions. Since we base our work on obtaining some meta-information from outside our type theory we call this the semantical method of formalising a productive function.

We restrict ourselves to the homographic algorithm, as the general case of accessible open expression is essentially similar but considerably more verbose. That is, instead of the general NAlg we consider the following restricted case which is the restriction of

\footnotetext{
${ }^{4}$ In fact it is the elimination principle of the inductive set which is the initial algebra of the functor $F(X)=1+(1+\mathbb{M}) \times X+(1+\mathbb{T}) \times X^{2}$, when considered as an inductive type.

${ }^{5}$ Intuitively, this is because an open expression tree is a finite composition of Möbius maps and tensors applied to a real number $x$. Composing $n$ Möbius maps and $m$ tensors (in one variable) results in a rational function with degree $2 n+m$.

${ }^{6}$ This is due to the fact that such expression trees are obtained from the continued fraction expansions of elementary functions [26, Section 10.2].
} 
NAlg on the open expression tree $\left\langle\left\langle\mu ; \perp_{[\mathbb{M} ; \mathbb{T}]}\right\rangle\right.$ :

$$
\mathrm{nf}_{\Phi}\left\langle\langle\mu ; \alpha\rangle:=\left\{\begin{array}{lll}
\varphi: \operatorname{nf}_{\Phi}\left\langle\left\langle\varphi^{-1} \circ \mu ; \alpha\right\rangle\right. & \text { if } \exists \varphi \in \Phi, & \operatorname{Incl}(\mu, \varphi), \\
\operatorname{nf}_{\Phi}\langle\langle\mu \circ \operatorname{hd}(\alpha) ; \mathrm{tl}(\alpha)\rangle & \text { otherwise. }
\end{array}\right.\right.
$$

Note that since $\alpha \in \Phi^{\omega}$ we could assume $\operatorname{nf}_{\Phi}(\alpha)=\alpha$ which eliminates the nested calls. Let $F_{\mathrm{H}}$ be the corresponding functional, i.e.,

$$
F_{\mathrm{H}}:=\lambda f:[\mathbb{M}] \rightarrow[\Phi] \lambda \theta:[\mathbb{M}] \begin{cases}\varphi: f\left(\varphi^{-1} \circ \operatorname{hd}(\theta): \mathrm{t}(\theta)\right) & \text { if } \quad \exists \varphi \in \Phi, \\ & \text { Incl }(\operatorname{hd}(\theta), \varphi), \\ f\left(\operatorname{hd}(\theta) \circ \operatorname{hd}(\mathrm{tl}(\theta)): \mathrm{t}^{2}(\theta)\right) & \text { otherwise. }\end{cases}
$$

Then we can prove that $\operatorname{cont}\left(F_{\mathrm{H}}, \mu: \alpha\right)$ holds [23, Lemma 5.6.1]. Since cont is an existential sentence, from this proof we can extract a function $\varkappa_{\mu: \alpha}: \mathbb{N} \longrightarrow \mathbb{N}$ such that (ignoring the subscript $\mu: \alpha$ for readability) for every $j$ we have that $\left(f^{\varkappa(j)}(\mu: \alpha)\right)_{j}$ is terminative (here as before $f^{k}$ is a shorthand for $F_{H}^{k}\left(\perp_{[\mathbb{M}] \longrightarrow[\Phi]}\right)$ ).

From here it is quite straightforward to obtain $\operatorname{nf}_{\Phi}\langle\langle\mu ; \alpha\rangle\rangle$ : in order to get the $j$ th element one has to take the $j$ th element of $F_{\mathrm{H}}^{\varkappa(j)}$.

We formalise this diagonalisation in MTT extended with coinductive types. First we define the iteration of $F_{\mathrm{H}}$. For this we need the coinductive type denoting $\left(\mathbb{M}^{\omega} \longrightarrow \Phi^{\omega}\right)^{\omega}$, i.e., the streams of functions from streams of matrices to streams of digits. This type can be introduced by using the $v$-FORMATION rule with the polynomial stream functor $\mathrm{F}_{\mathrm{A}}(X)=$ $A \times X$ three times. First by taking $A=\Phi$ and $A=\mathbb{M}$ and using $\nu \mathrm{F}_{\Phi}$-Formation and $\nu \mathrm{F}_{\mathbb{M}}$-FORMATION we form the coinductive types denoting $\Phi^{\omega}$ and $\mathbb{M}^{\omega}$. Consequently, by taking $A=\mathbb{M}^{\omega} \longrightarrow \Phi^{\omega}$ we form the coinductive type denoting $\left(\mathbb{M}^{\omega} \longrightarrow \Phi^{\omega}\right)^{\omega}$.

After this we use $\nu \mathrm{F}_{\mathbb{M}^{\omega}} \longrightarrow \Phi^{\omega}$-INTRODUCTION to obtain an element of this type:

$$
\frac{\mathbb{M}^{\omega} \longrightarrow \Phi^{\omega}: s \quad x: \mathbb{M}^{\omega} \longrightarrow \Phi^{\omega} \vdash\left\langle F_{\mathrm{H}}(x), F_{\mathrm{H}}(x)\right\rangle: \mathrm{F}_{\mathbb{M}^{\omega}} \longrightarrow \Phi^{\omega}\left(\mathbb{M}^{\omega} \longrightarrow \Phi^{\omega}\right)}{g: \mathbb{M}^{\omega} \longrightarrow \Phi^{\omega} \vdash\left(\nu-\text { it } \quad \lambda x \cdot\left\langle F_{\mathrm{H}}(x), F_{\mathrm{H}}(x)\right\rangle \quad g\right):\left(\mathbb{M}^{\omega} \longrightarrow \Phi^{\omega}\right)^{\omega}} .
$$

If we denote $\left(\nu-\right.$ it $\left.\lambda x \cdot\left\langle F_{\mathrm{H}}(x), F_{\mathrm{H}}(x)\right\rangle\right)$ by iter_ $\mathrm{F}_{\mathrm{H}}$ then using $\nu \mathrm{F}_{\mathbb{M}^{\omega}} \rightarrow \Phi^{\omega}$-EQUALITY we can derive the fixpoint equation of iter $F_{\mathrm{H}}$, namely

$$
\text { iter_ } \mathrm{F}_{\mathrm{H}} \quad g=F_{\mathrm{H}} \quad g: \text { iter_}_{-} \mathrm{F}_{\mathrm{H}}\left(F_{\mathrm{H}} g\right) \text {. }
$$

For taking the diagonal we need an auxiliary function which is definable in MTT using structural recursion. This is the function $\mathrm{nth}_{A}: A^{\omega} \times \mathbb{N} \longrightarrow A$ that given a stream $\alpha \in A^{\omega}$ and a natural number $n \geq 0$ returns $\alpha(n)$, i.e., the $n$th element of $\alpha$. The recursive declaration for $n$th is

$$
\mathrm{nth}_{A}\langle\alpha, n\rangle:= \begin{cases}\operatorname{hd}(\alpha) & \text { if } n=0 \\ \operatorname{nth}_{A}\langle\mathrm{tl}(\alpha), n-1\rangle & \text { otherwise. }\end{cases}
$$

We assume ${ }^{7}$ that $\mathrm{nth}_{A}$ is defined for every $A$.

\footnotetext{
${ }^{7}$ To be precise, to define nth one has to apply $\nu \mathrm{F}_{\mathrm{A}}$-FORMATION and subsequently the $W$-type constructor of MTT [19], [25] to formalise the recursive declaration of $n$ th.
} 
In what follows let $\pi_{i}(1 \leq i \leq 4)$ denote the $i$ th projection of a quadruple. We use $\nu \mathrm{F}_{\Phi}$-INTRODUCTION to take the diagonal of the iteration of $F_{\mathrm{H}}$ :

$$
\begin{gathered}
(A:=) \quad\left(\mathbb{M}^{\omega} \longrightarrow \Phi^{\omega}\right)^{\omega} \times \Phi^{\omega} \times \mathbb{N}^{\mathbb{N}} \times \mathbb{N}: s, \\
\begin{array}{cc}
a: A \vdash(B(a):=) \quad & \left\langle\mathrm{nth}_{\Phi}\left\langle\left(\mathrm{nth}_{\mathbb{M}^{\omega}} \longrightarrow \Phi^{\omega}\left\langle\pi_{1}(a), \pi_{3}(a)\left(\pi_{4}(a)\right)\right\rangle\right) \circ \pi_{2}(a), \pi_{4}(a)\right\rangle,\right. \\
& \left.\left\langle\pi_{1}(a), \pi_{2}(a), \pi_{3}(a), \pi_{4}(a)+1\right\rangle\right): \mathrm{F}_{\Phi}(A)
\end{array} \\
c: A \vdash(v-\text { it } \lambda x \cdot B(x) \quad c): \Phi^{\omega}
\end{gathered}
$$

If we denote ( $\nu$-it $\lambda x \cdot B(x)$ ) by diagonal then using $\nu \mathrm{F}_{\Phi}$-EQUALITY we can derive the fixpoint equation of diagonal, namely

$$
\begin{aligned}
& \text { diagonal } \quad X S \quad x s \quad g \quad n=\mathrm{nth}_{\Phi}\left\langle\left(\mathrm{nth}_{\mathbb{M}^{\omega}} \longrightarrow \Phi^{\omega}\langle X S, g(n)\rangle\right) \quad x s, n\right\rangle \text { : } \\
& \text { diagonal } \quad X S \text { xs } \quad g \quad n+1 \text {. }
\end{aligned}
$$

To define the homographic algorithm according to the dcpo semantics, we need to iterate $F_{\mathrm{H}}$ on $\perp_{[\mathrm{M}]} \longrightarrow[\Phi]$. What this means is that we have to start from no information and by iterating $F_{\mathrm{H}}$ incrementally obtain more information. Therefore, in MTT, we can also iterate $F_{\mathrm{H}}$ on an arbitrary element of $\mathbb{M}^{\omega} \longrightarrow \Phi^{\omega}$. Thus assuming $\varphi \in \Phi$ we consider $\varphi^{\omega} \in \Phi^{\omega}$ which is the stream containing only the digit $\varphi$. We first show that $\varphi^{\omega}$ can be constructed using $\nu \mathrm{F}_{\Phi}$-INTRODUCTION and the singleton set 1as follows: ${ }^{8}$

$$
\frac{\mathbf{1}: s \quad x: \mathbf{1} \vdash\langle\varphi, x\rangle: \Phi \times \mathbf{1}}{y: \mathbf{1} \vdash(\nu-\text { it } \quad \lambda x \cdot\langle\varphi, x\rangle \quad y): \Phi^{\omega}} .
$$

If we denote ( $\nu$-it $\lambda x \cdot\langle\varphi, x\rangle \quad y$ ) by phis, once again using $\nu \mathrm{F}_{\Phi}$-EQUALITY we can derive the fixpoint equation of phis, which is

$$
\text { phis }=\varphi \text { : phis. }
$$

Extensionally we know that phisis the same as $\varphi^{\omega}$, since this is provable in a categorical model of MTT where we can use the uniqueness property of the final coalgebra.

Finally we are ready to define the homographic algorithm in terms of iter_ $F_{H}$, diagonaland phisand assuming the existence of $\varkappa$ :

$$
\mathrm{H} \quad x s:=\text { diagonal } \quad\left(\text { iter_ } \mathrm{F}_{\mathrm{H}} \quad \lambda x: \mathbb{M}^{\omega} \cdot \text { phis) } \quad x s \quad \varkappa(x s) \quad 0 .\right.
$$

Thus we have formalised the homographic algorithm in MTT extended with coinductive types.

The above method which relies on the existence of the function $\varkappa$ can be generalised for formalising $\mathrm{nf}_{\Phi}$ on arbitrary accessible open expression trees. This is because by Proposition 6.13 we are able to find a modulus function $\varkappa$. The rest of the technique is similar to what we did above for the homographic case.

Following a method similar to above, it is also possible to extract a function $\varkappa^{\prime}: \mathbb{E} \longrightarrow \Phi \times \mathbb{E}$ that, given an expression tree $\theta$, outputs a digit $\varphi_{i}$ and an expression

\footnotetext{
${ }^{8}$ Type 1 of a singleton set can be constructed by the rules for enumeration sets in MTT [25, p. 44]. So here we assume its existence.
} 
tree $\theta^{\prime}$. The digit $\varphi_{i}$ is the next digit that would be emitted by NAlg with the input $\theta$, and $\theta^{\prime}$ would be the rest of the input (or the new input) after emitting $\varphi_{i}$. Subsequently, the function $\varkappa^{\prime}$ can be used to rewrite $\mathrm{nf}_{\Phi}$ as

$$
\mathrm{nf}_{\Phi}(\theta):=\psi: \mathrm{nf}_{\Phi}\left(\theta^{\prime}\right) \quad \text { where }\left\langle\psi, \theta^{\prime}\right\rangle=\varkappa^{\prime}[\theta] .
$$

Again - as was the case with diagonal and iter_ $F_{H}$ above - this shape of NAlg can be generated using the $\nu$-INTRODUCTION rule. That is to say, for $\theta \in \mathbb{E}$ we define $\operatorname{NAlg}(\theta):=$ $\left(\begin{array}{lll}\nu \text {-it } & \varkappa^{\prime} & \theta\end{array}\right)$.

One can prove the extensional fixpoint equation of $\mathrm{H}$, i.e., the fact that the two sides of (7.1) are pointwise equal streams. However, intensionally the fixpoint equation does not hold as the above definition and the definition in (7.1) are not convertible. This shows that in order to prove further properties of $\mathrm{H}$ (i.e., correctness) one has to work with the bisimulation equivalence and in a setoid of streams and expression trees.

Note that in the present paper we have only dealt with formalising NAlg in type theory. That means we tried to write it in the language of our type theory. After formalising the algorithm we need to verify its correctness. In addition to the use of bisimulation, this involves proving that the formalised algorithm satisfies its mathematical specifications. In the case of NAlg we should prove that for the various choices of families of expression trees as given in [26], this algorithm computes the respective elementary function. This part of the work, although mathematical in nature, involves some type theoretic issues and deserves separate treatment.

\section{Analysis of the Method}

Although our focus was on the normalisation algorithm of exact arithmetic, the method that we used in the previous sections can be applied in other instances of dealing with the general recursive function on infinite objects. Suppose $A$ and $D$ are two infinite objects. In order to formalise $f: D \longrightarrow A$ which is given in terms of a specification in a lazy functional programming language, one first has to formalise $A$ and $D$ as a coinductive type using the rules of Section 2. Subsequently, one has to impose a dcpo structure on $D$ and $A$ for which maximal elements are productive elements. This will enable us to state and prove the productivity of the fixpoint of functional $F:=\lambda f \lambda x ; f x$. Implicit in this proof is an existential quantifier that gives us the modulus of convergence with respect to the dcpo order that we have put on $D$ and $A$. Note that such an order is essential for specifying what the "initial segments" of input and output are.

Finally, one can use the modulus of convergence as in Section 7 to formalise a function which is extensionally equal to the original specification of $f$. Note that the definition of diagonal is independent of the definition of $F_{\mathrm{H}}$; moreover, the definition of iter_ $\mathrm{F}_{\mathrm{H}}$ can be parametrised in terms of $F_{\mathrm{H}}$ (by adding an extra argument). Thus the method in Section 7 can be easily adjusted to be applied to the general case. Such a treatment is done in detail in Section 4.7 of [23] for a large class of filter-like specifications. It remains to be seen what portion of this method is mechanisable.

We emphasise that in this article the proof that we formalise in MTT is not the proof of productivity in terms of dcpo's. We use the dcpo-based proof to extract the modulus of convergence $\varkappa$ which can then be internalised in our type theory. However, it is possible 
to formalise the proof of productivity in type theory too; but we do not consider it in this paper. In fact our method is similar to the method of [2] for inductive types.

We based our work on the Knaster-Tarski fixpoint theorem by taking the fixpoint on the dcpo of continuous functions. An alternative method would be to use the Banach fixpoint theorem and instead of productivity prove the contractivity of the functionals. This is the approach taken by Matthews [20] and Di Gianantonio and Miculan [8] for formalising general recursive functions on coinductive types. Restricting to the normalisation algorithm, Potts et al. [27] use an altogether different approach, by working in an extension of PCF and using the power-domain of intervals with rational endpoints.

Although our notion of productivity and the proofs based upon it might be somehow tedious, the idea behind the proofs and the intuition behind the definition of productivity remains straightforward. Formalisation of the above alternative approaches will not lead to less tedious proofs because to prove the productivity one ought to capture the metric properties of the representation, the matrices and the tensors one way or another. In the Di Gianantonio-Miculan method this is dealt with within the proof of the contractivity modulo an equivalence relation. While Potts uses power-domains which are type theoretically more complex structures than our simple dcpo's.

\section{NAlg with a Postponed Productivity}

The extension of MTT that we introduced in Section 2 was a very simple extension whose main purpose was to accommodate lazy streams and expression trees. This simplicity might seem to be the reason why we had to adhere to semantic approaches to formalise NAlg. In some sense this is true: the type theory should be ensured of the productivity of an infinite object before it accepts the infinite object. If there is no syntactical way of ensuring the productivity then one has to use semantical methods. This semantic information can then be presented as a proof obligation to formalise an infinite object in type theory. A proof obligation is a proposition asserting that the function is productive and will be considered as an extra argument to the function [6]. In the formalisation of Section 7 the proof obligation is present in the form of the existence of the function $\varkappa$.

Bearing in mind that NAlg is a partial function, one way or another we have to enter the proof obligation into the picture. However, if we use a more delicate extension of MTT with coinductive types we might be able to postpone our dependence to the semantic proofs such as the ones in Section 6. One such type theory that inhabits very complicated coinductive types is the Calculus of Inductive Constriction (CIC) extended with coinductive types [16] which is implemented as the Coq proof assistant [7].

We explain what we mean by postponing the dependence to semantic proofs in CIC. In CIC as in any other constructive type theory, all the functions should be total. The only way to formalise a partial function is to change either the domain or the codomain of the function and formalise an extensionally equal function. One approach to formalising partial functions is the inductive domain predicate which is developed for formalising partial or nonstructurally recursive functions while still keeping the general shape (and hence complexity) of the function intact [9], [4]. According to this method one can define the proof obligation to be an inductively defined predicate. This will allow us to define the function easily without knowing whether it is partial or total. Furthermore, it allows 
for computing (inside Coq) with those elements of the domain for which we know-i.e., we have a proof-that they are in the domain. In our case if we could apply this method we would be able to compute with NAlg on specific expression trees without having to prove a general result about the productivity on a family of expression trees and hence postponing the semantic arguments.

The above idea of using inductive domain predicates was originally proposed for recursive functions. Recent work by Bertot [3] shows how to apply this method to formalise partial functions on coinductive types. The author has devised a method similar to Bertot's method, which employ the inductive domain predicate paradigm for formalising the homographic and quadratic algorithms in $\operatorname{Coq}$ [24]. For the general case of NAlg one has to take into account the nestedness in the definition of NAlg. ${ }^{9}$ This is the subject of further study by the author.

The inductive domain predicate method needs the type theory to be enriched with much more complex coinductive types than only the simple polynomial functors and hence is not applicable to the simple extension of MTT that we gave in Section 2. The method is heavily based on the use of dependent and polymorphic coinductive types. These are available in CIC as well as in some other extensions of MTT with coinductive types, namely those in [17], [22].

Obviously, since we worked in a type theory which is simpler than CIC we can formalise the coinductive objects of this paper in Coq. However, we have to keep in mind that in CIC the equality (or conversion) rule of coinductive types is different from $v$ F-EQUALITY. In fact CIC uses a cofixed-point expansion rule that allows for the expansion of a cofixed-point -i.e., a coinductively defined term-only when a case analysis of the cofixed-point is done. However, the usual coiteration schemes can be derived in terms of cofixed-points [15] and therefore our $v$ F-EQUALITY is compatible with cofixed-point expansion in Coq.

Practically, when we are using the proof assistant Coq, the modification of NAlg using the function $\varkappa$ will also help in satisfying the guardedness condition. This condition is a syntactic criterion added to the $C o q$ type checker in order to ensure the productivity of infinite objects [16]. The guardedness condition is not very powerful and can only be used for formalising infinite objects that have a canonical shape: the recursive occurrence of the infinite object is the immediate (i.e., unguarded) argument of the constructor of the coinductive type of the codomain. Fortunately, the function $\varkappa$ enables us to satisfy this condition and hence we can formalise the function $\mathrm{H}$ of Section 7 in Coq. Such a formalisation is given in the Appendix.

\section{Conclusions and Further Work}

We have shown that the normalisation algorithm of Edalat and Potts can be formalised in type theory if some semantical information concerning its productivity is available. Such information is obtained from the topological properties of the real number representation and Möbius maps and tensors. We showed that we can prove the productivity

\footnotetext{
${ }^{9}$ The combination of the nestedness and inductive domain predicate approach leads to the use of induction-recursion [10] which is not available in Coq but one can simulate it in Coq.
} 
in a semantical model outside type theory. After that we can formalise the NAlg inside type theory by using the meta information which exists in the form of a modulus of convergence function. As we showed, since we have put the burden of productivity on a semantic model, the machinery of coinductive types added to our type theory need not be very complex. In fact only having coinductive types of a simple class of functors suffices.

Several extensions to the present work are imaginable. The most important followup to this work would be to formalise the proof of correctness of (special cases) of NAlg. Note that this is the main motivation behind formalising algorithms in constructive type theory. As is evident from the proof of correctness of the homographic algorithm in Corollary 5.6.15 of [23], once we have proven the productivity proving the correctness is less tedious. Therefore, formalising the proof of correctness of the accessible open expression trees is the next step in this work. This will lead to the correctness of a lazy algorithm for evaluating rational functions.

After that one has to formalise the productivity and correctness of NAlg on larger families of expression trees such as those used by Potts to evaluate elementary real functions. First one should try to extend Proposition 6.13 to include the families of expression trees as large as possible. This is closely related to assessing the strength of the notion of $\mathbb{E}$-computability which was introduced in Section 5.6 of [23].

As pointed out earlier, parallel to this work the author has considered the more syntactical approach of inductive domain predicates for formalising partial function. First results concerning a field structure on coalgebra of real numbers are presented in [24]. Further ongoing work involves tackling the nested branches of NAlg by studying a dual notion to Dybjer's induction-recursion [10] for coinductive types, and exploring ways to simulate induction-recursion in Coq.

\section{Acknowledgment}

The author thanks an anonymous referee for insightful remarks and corrections that helped improve the article.

\section{Appendix. Coq Formalisation of Semantical Method}

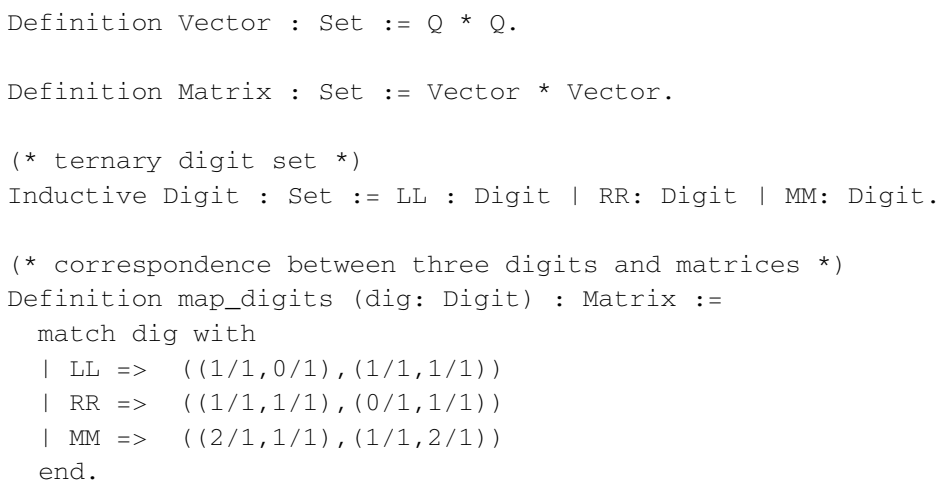




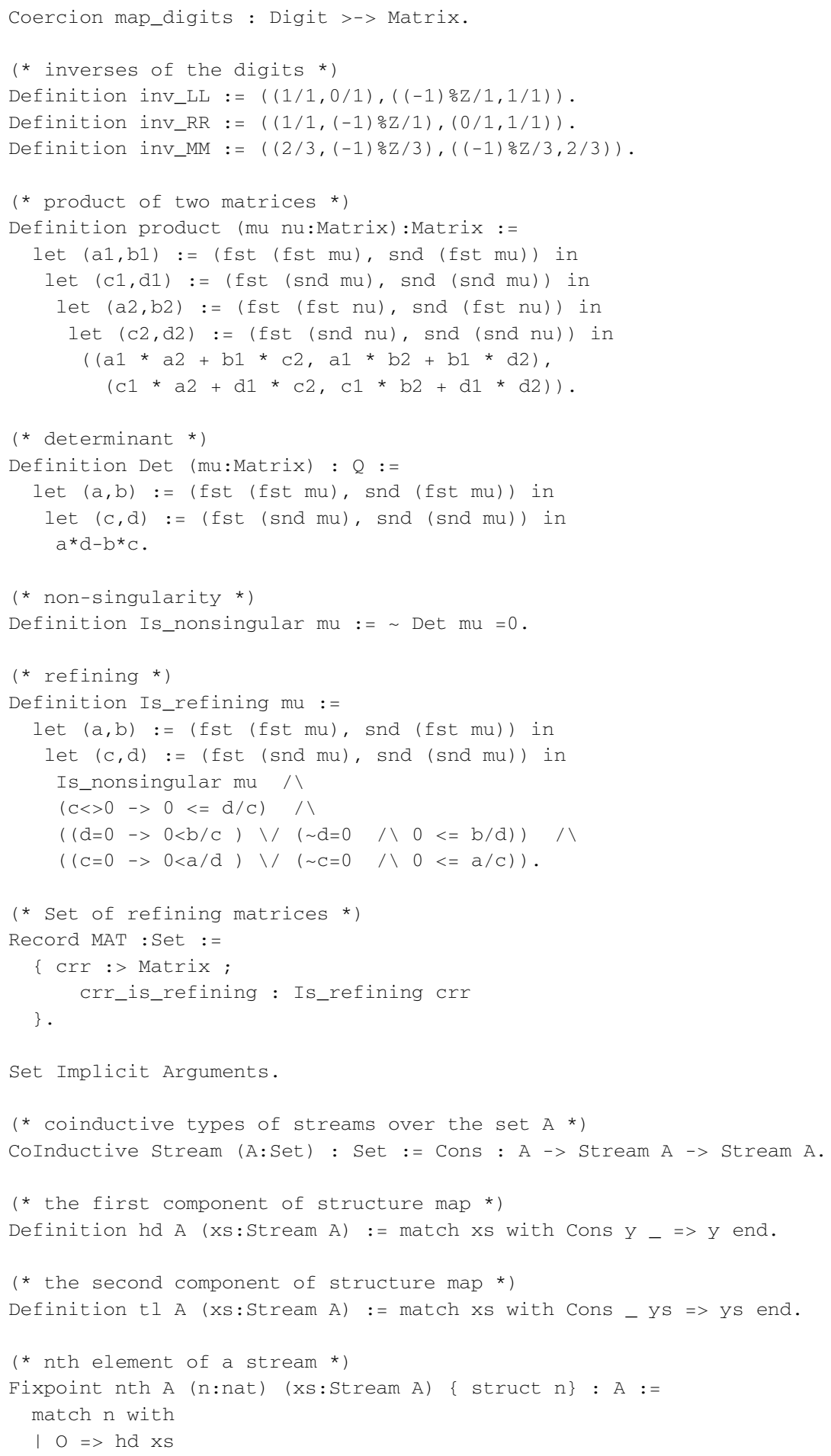




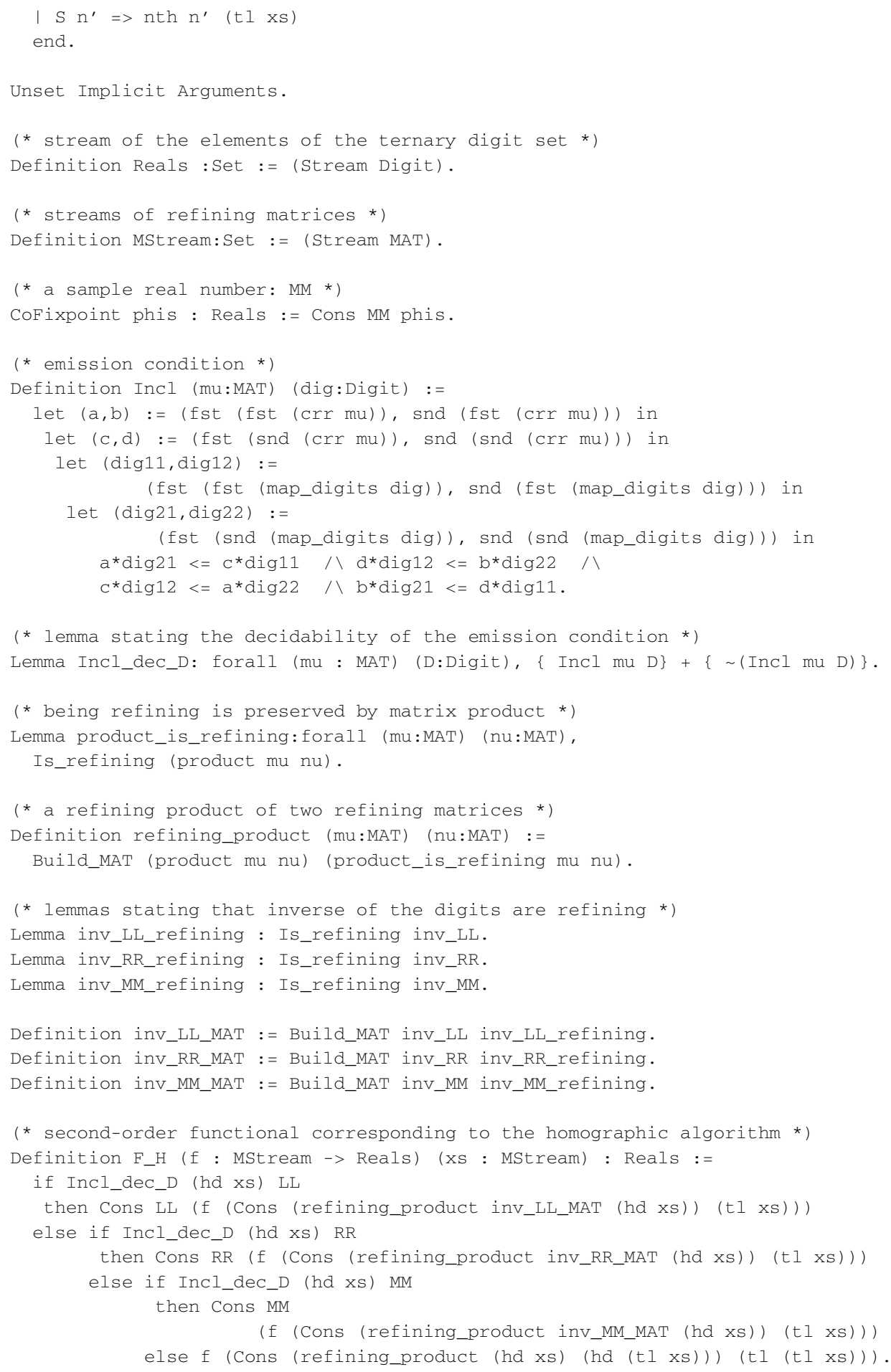




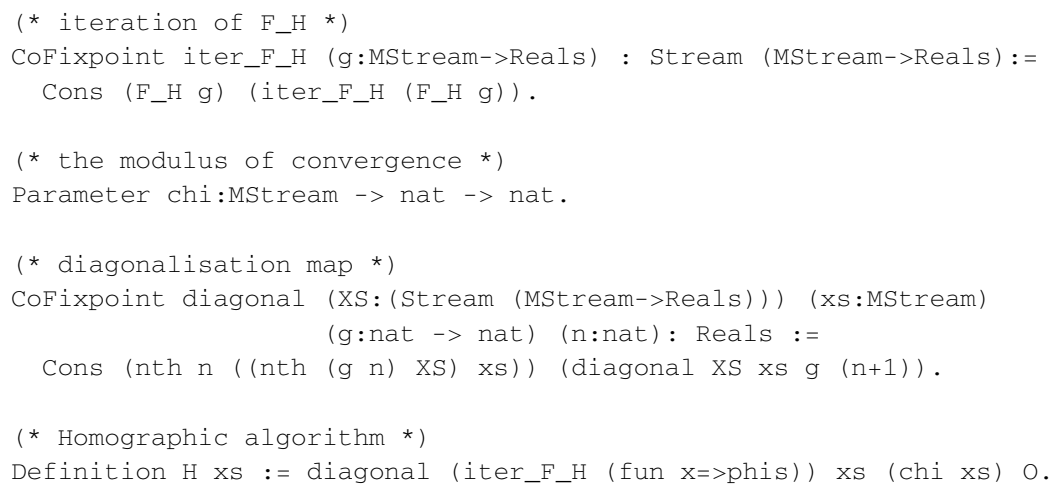

\section{References}

[1] S. Abramsky and A. Jung. Domain theory. In S. Abramsky, D. M. Gabbay, and T. S. E. Maibaum, editors, Handbook of Logic in Computer Science, volume 3, pages 1-168. Clarendon Press, Oxford, 1994.

[2] A. Balaa and Y. Bertot. Fonctions récursives générales par itération en théorie des types. In Proceedings des 13èmes Journées francophones des langages applicatifs. INRIA, 2002.

[3] Y. Bertot. Filters on coinductive streams, an application to Eratosthenes' sieve. In P. Urzyczyn, editor, TLCA, pages 102-115. Volume 3461 of Lecture Notes in Computer Science. Springer-Verlag, Berlin, 2005.

[4] A. Bove and V. Capretta. Nested general recursion and partiality in type theory. In R. J. Boulton and P. B. Jackson, editors, Theorem Proving in Higher Order Logics, 14th International Conference, TPHOLs 2001, Edinburgh, Scotland, UK, September 3-6, 2001, Proceedings, pages 121-135. Volume 2152 of Lecture Notes in Computer Science. Springer-Verlag, Berlin, 2001.

[5] V. Capretta. Abstraction and Computation. Ph.D. thesis, Katholieke Universiteit Nijmegen, Apr. 2002.

[6] J. Carlström. Subsets, quotients and partial functions in Martin-Löf's type theory. In [14], pages 78-94.

[7] The Coq Development Team. The Coq Proof Assistant Reference Manual, Version 8.0. LogiCal Project, Apr. 2004. http: / / coq. inria. fr/doc/main.html [cited 15 March 2006].

[8] P. Di Gianantonio and M. Miculan. A unifying approach to recursive and co-recursive definitions. In [14], pages 148-161.

[9] C. Dubois and V. V. Donzeau-Gouge. A step towards the mechanization of partial functions: domains as inductive predicates. In M. Kerber, editor, Proceedings of the Workshop on Mechanization of Partial Functions, July 5 1998, Lindau, Germany, pages 53-62, 1998. Available at ftp: / / ftp.cs.bham.ac.uk/pub/authors/M.Kerber/98-CADE-WS/dubois-donzeau . ps.gz [cited 15 March 2006].

[10] P. Dybjer. A general formulation of simultaneous inductive-recursive definitions in type theory. J. Symbolic Logic, 65(2):525-549, 2000.

[11] A. Edalat and R. Heckmann. Computing with real numbers. In G. Barthe, P. Dybjer, L. Pinto, and J. Saraiva, editors, Applied Semantics: Advanced Lectures, pages 193-267. Volume 2395 of Lecture Notes in Computer Science. Springer-Verlag, Berlin, 2002.

[12] A. Edalat and P. J. Potts. A new representation for exact real numbers. In S. Brookes and M. Mislove, editors, Mathematical Foundations of Programming Semantics, Thirteenth Annual Conference (MFPS XIII), Carnegie Mellon University, Pittsburgh, PA, USA, March 23-26, 1997, pages 119-132. Volume 6 of Electronic Notes in Theoretical Computer Science. Elsevier, Amsterdam, 1997.

[13] A. Edalat, P. J. Potts, and P. Sünderhauf. Lazy computation with exact real numbers. In M. Berman and S. Berman, editors, Proceedings of the Third ACM SIGPLAN International Conference on Functional Programming (ICFP-98), Baltimore, Maryland, USA, September 27-29, 1998, pages 185-194. Volume 34(1) of ACM SIGPLAN Notices. ACM Press, New York, 1999. 
[14] H. Geuvers and F. Wiedijk, editors. Types for Proofs and Programs: International Workshop, TYPES 2002, Berg en Dal, The Netherlands, April 24-28, 2002. Selected Papers. Volume 2646 of Lecture Notes in Computer Science. Springer-Verlag, Berlin, 2003.

[15] E. Giménez. Codifying guarded definitions with recursive schemes. In P. Dybjer, B. Nordström, and J. Smith, editors, Types for Proofs and Programs, International Workshop TYPES'94, Båstad, Sweden, June 6-10, 1994, Selected Papers, pages 39-59. Volume 996 of Lecture Notes in Computer Science. Springer-Verlag, Berlin, 1995.

[16] E. Giménez. Un Calcul de Constructions Infinies et son Application a la Verification des Systemes Communicants. Ph.D. thesis, Laboratoire de l'Informatique du Parallélisme, Ecole Normale Supérieure de Lyon, Dec. 1996.

[17] P. Hancock and A. Setzer. Interactive programs and weakly final coalgebras in dependent type theory. In L. Crosilla and P. Schuster, editors, From Sets and Types to Topology and Analysis. Towards Practicable Foundations for Constructive Mathematics, Proceedings of the Workshop, 12-16 May 2003, Venice International University, San Servolo, Venice, Italy, pages 115-134. Volume 48 of Oxford Logic Guides. Oxford University Press, New York, 2005.

[18] R. Heckmann. Contractivity of linear fractional transformations. Theoret. Comput. Sci., 279(1-2):6582, May 2002

[19] P. Martin-Löf. Intuitionistic Type Theory. Biblioplois, Napoli, 1984. Notes of Giovanni Sambin on a series of lectures given in Padova.

[20] J. Matthews. Recursive function definition over coinductive types. In Y. Bertot, G. Dowek, A. Hirschowitz, C. Paulin, and L. Théry, editors, Theorem Proving in Higher Order Logics, 12th International Conference, TPHOLs'99, Nice, France, September, 1999, Proceedings, pages 73-90. Volume 1690 of Lecture Notes in Computer Science. Springer-Verlag, Berlin, 1999.

[21] N. P. Mendler, P. Panangaden, and R. L. Constable. Infinite objects in type theory. In Symposium on Logic in Computer Science (LICS '86), Washington, D.C., USA, June 1986, pages 249-255. IEEE Computer Society Press, Los Alamitos, CA, 1986.

[22] M. Michelbrink and A. Setzer. State dependent IO-monads in type theory. In L. Birkedal, editor, Proceedings of the 10th Conference on Category Theory in Computer Science (CTCS 2004), pages 127-146. Volume 122 of Electronic Notes in Theoretical Computer Science. Elsevier, Amsterdam, Mar. 2005.

[23] M. Niqui. Formalising Exact Arithmetic: Representations, Algorithms and Proofs. Ph.D. thesis, Radboud Universiteit Nijmegen, Sept. 2004.

[24] M. Niqui. Coinductive field of exact real numbers and general corecursion. In N. Ghani and J. Power, editors, Proceedings of the 8th International Workshop on Coalgebraic Methods in Computer Science (CMCS 2006), pages 121-139. Volume 164.1 of Electronic Notes in Theoretical Computer Science. Elsevier, Amsterdam, Oct. 2006.

[25] B. Nordström, K. Peterson, and J. M. Smith. Programming in Martin-Lof's Type Theory. Volume 7 of International Series of Monographs on Computer Science. Oxford University Press, New York, 1990.

[26] P. J. Potts. Exact Real Arithmetic Using Möbius Transformations. Ph.D. thesis, University of London, Imperial College, London, July 1998.

[27] P. J. Potts, A. Edalat, and M. H. Escardó. Semantics of exact real number arithmetic. In Proceedings of the 12th Annual IEEE Symposium on Logic in Computer Science, pages 248-257, 1997.

[28] J. J. M. M. Rutten. Universal coalgebra: a theory of systems. Theoret. Comput. Sci., 249(1):3-80, Oct. 2000.

[29] A. Saïbi. Typing algorithm in type theory with inheritance. In POPL '97: Proceedings of the 24th ACM SIGPLAN-SIGACT Symposium on Principles of Programming Languages, pages 292-301. ACM Press, New York, 1997.

[30] B. A. Sijtsma. On the productivity of recursive list definitions. ACM Trans. Program. Lang. Syst. (TOPLAS), 11(4):633-649, Oct. 1989.

[31] K. Weihrauch. Computable Analysis. An Introduction. Springer-Verlag, Berlin, 2000.

Received September 2, 2005, and in final form April 10, 2006. Online publication April 20, 2007. 